\title{
Superspace formulation of general massive gauge theories and geometric interpretation of mass-dependent BRST symmetries
}

\author{
B. GEYer円 \\ Universität Leipzig, Naturwissenschaftlich-Theoretisches Zentrum and \\ Institut für Theoretische Physik, 04109 Leipzig, Germany \\ D. MÜLSCH \\ Wissenschaftszentrum Leipzig e.V., Leipzig 04103, Germany
}

\begin{abstract}
A superspace formulation is proposed for the $\operatorname{osp}(1,2)$-covariant Lagrangian quantization of general massive gauge theories. The superalgebra $\operatorname{osp}(1,2)$ is considered as subalgebra of the superalgebra $\operatorname{sl}(1,2) \sim \operatorname{osp}(2,2)$ which may be considered as the algebra of generators of the conformal group in a superspace with two anticommuting coordinates. The mass-dependent (anti)BRST symmetries of proper solutions of the quantum master equations in the osp $(1,2)$-covariant formalism are realized in that superspace as invariance under translations combined with mass-dependent special conformal transformations. The $S p(2)$ symmetry - in particular the ghost number conservation - and the new ghost number conservation are realized in the superspace as invariance under symplectic rotations and dilatations, respectively. The new ghost number conservation is generally broken by the choice of a gauge. The transformations of the gauge fields and the full set of necessarily required (anti)ghost and auxiliary fields under the superalgebra $\operatorname{sl}(1,2)$ are determined both for irreducible and first-stage reducible theories with closed gauge algebra.
\end{abstract}

\footnotetext{
${ }^{1}$ e-mail: geyer@itp.uni-leipzig.de
} 


\section{Introduction}

After the realization that the effective Lagrangian of non-abelian gauge theories is invariant with respect to Becchi-Rouet-Stora-Tyutin (BRST) [1] as well as anti-BRST transformations [2], it has been recognized that this invariance can be used as a fundamental principle in the construction of covariantly quantized gauge theories (for a modern introduction see [3]). In particular, a superfield formulation of quantized pure Yang-Mills theories by Bonora and Tonin provides a convenient framework for describing the extended BRST symmetries [4]. In this framework the extended BRST symmetries are realized as translations in a superspace along additional anticommuting coordinates (for a more recent approach, we refer to [5] and references therein).

A $S p(2)$-covariant superfield description of Lagrangian quantization of general gauge theories, which is applicable irrespective of whether the theories are irreducible or reducible and whether the gauge algebra is closed or open, has been given in Ref. [6]. A corresponding superfield formulation of the quantization procedure in the Hamiltonian approach for theories with first-class constraints has been given in Ref. [7].

Recently, the $S p(2)$-quantization of Batalin, Lavrov and Tyutin(BLT) has been extended to a formalism which is based on the orthosymplectic superalgebra $\operatorname{osp}(1,2)$ [8] and which can be applied to massive gauge theories. This is achieved by incorporating into the extended BRST transformations $m$-dependent terms in such a way that the $m-$ extended (anti)BRST symmetry of the quantum action $W_{m}$ is preserved. In that approach $W_{m}$ is required to satisfy the generalized quantum master equations of $m$-extended BRST symmetry and, in addition, of $S p(2)$ symmetry,

$$
\begin{aligned}
& \frac{1}{2}\left(W_{m}, W_{m}\right)^{a}+V_{m}^{a} W_{m}=i \hbar \Delta^{a} W_{m} \quad \Longleftrightarrow \quad \bar{\Delta}^{a} \exp \left\{W_{m}\right\}=0, \\
& \frac{1}{2}\left\{W_{m}, W_{m}\right\}_{\alpha}+V_{\alpha} W_{m}=i \hbar \Delta_{\alpha} W_{m} \Longleftrightarrow \bar{\Delta}_{\alpha} \exp \left\{W_{m}\right\}=0,
\end{aligned}
$$

respectively, whose generating (second order) differential operators

$$
\begin{aligned}
\bar{\Delta}_{m}^{a} & \equiv \Delta^{a}+(i / \hbar) V_{m}^{a}, & & (a=1,2), \\
\bar{\Delta}_{\alpha} & \equiv \Delta_{\alpha}+(i / \hbar) V_{\alpha}, & & (\alpha=0, \pm 1),
\end{aligned}
$$

form a superalgebra isomorphic to $\operatorname{osp}(1,2)$ (the definitions of the (anti)brackets and the operators $\bar{\Delta}_{m}^{a}$ and $\bar{\Delta}_{\alpha}$ are given below).

The incorporation of mass terms into the action $W_{m}$ is necessary at least intermediately in the renormalization scheme of Bogoliubov, Parasiuk, Hepp, Zimmermann and Lowenstein(BPHZL) 9]. An essential ingredient to deal with massless theories in that 
scheme consists in the introduction of a regularizing mass $m=(s-1) M$ for any massless field and performing ultraviolet as well as infrared subtractions thereby avoiding spurious infrared singularities in the limit $s \rightarrow 1$. By using such an infrared regularization without violating the extended BRST symmetries - the $\operatorname{osp}(1,2)$-superalgebra appears necessarily.

Moreover, the BPHZL renormalization scheme is probably the mathematical best founded one in order to formulate the quantum master equations on the level of algebraic renormalization theory and to properly compute higher-loop anomalies [10]. The reason is the following: The only quantity that remains undefined in the above mentioned approaches of quantizing general gauge theories is the right-hand side of the quantum master equations (that problem already occurs in the Batalin-Vilkovisky(BV) field-antifield formalism). At the classical level, the extended BRST invariance in the osp (1,2)-approach is expressed by the classical master equations $\frac{1}{2}\left(S_{m}, S_{m}\right)^{a}+V_{m}^{a} S_{m}=0$, where $S_{m}$ is the lowest order approximation in $\hbar$ of $W_{m}$. On the quantum level, formal manipulations modify the classical master equations into Eq. (1.1). When applied to the local functional $W_{m}$ the operation $\Delta^{a} W_{m}$ leads to the ill-defined expression $\delta(0)$. Well-defined expressions for the regularized operators $\Delta^{a}$ are proposed at one-loop level in 11] within the context of Pauli-Villars regularization and at higher order in [12 for non-local regularization. However, by means of the BPHZL renormalization scheme, which bypasses any ultraviolet regularizations, the right-hand side of the quantum master equations can be defined by using Zimmermanns's normal products to any order of perturbation theory 10 .

The purpose of the present paper is to reveal the geometrical content of the osp $(1,2)-$ covariant Lagrangian quantization which amounts to understand the geometrical meaning of the $m$-dependent part of the extended BRST transformations. For that reason the theory will be described in terms of super(anti)fields. Our approach is based on the idea to consider $\operatorname{osp}(1,2)$ as subsuperalgebra of the superalgebra $\operatorname{sl}(1,2)$. The latter algebra, being isomorphic to $\operatorname{osp}(2,2)$, contains four bosonic generators $V_{\alpha}$ and $V$, which form the Lie algebra $\operatorname{sl}(2) \oplus u(1)$, and four (nilpotent) fermionic generators $V_{+}^{a}$ and $V_{-}^{a}$. The even part of $\operatorname{sep}(1,2)$ is the algebra $s l(2)$ generating the special linear transformations, but due to their isomorphism to the algebra $s p(2)$ we will speak about symplectic transformations. The eigenvalues of the generators $V_{\alpha}$ for $\alpha=0$ define the ghost numbers, whereas the eigenvalues of the generator $V$ define what in Ref. [13] was called the 'new ghost number'. The generators $V_{+}^{a}$ and $V_{-}^{a}$ have opposite new ghost numbers, $\operatorname{ngh}\left(V_{ \pm}^{a}\right)= \pm 1$, respectively. 
But, introducing a mass $m$ which formally will be attributed also by a new ghost number, $\operatorname{ngh}(m)=1$, they can be combined into two fermionic generators $V_{m}^{a}=V_{+}^{a}+\frac{1}{2} m^{2} V_{-}^{a}$ of the superalgebra $\operatorname{osp}(1,2)$. For $m \neq 0$ these generators $V_{m}^{a}$ are neither nilpotent nor do they anticommutate among themselves.

The key observation that allows for a geometric interpretation of the superalgebra $\operatorname{sl}(1,2)$ is due to Baulieu, Siegel and Zwiebach [14] which in a quite different context of string theory gave a description of $\operatorname{sl}(1,2)$ as the algebra generating conformal transformations in a 2-dimensional superspace. Hence, the generators $V_{+}^{a}, V_{-}^{a}, V^{a b}=\left(\sigma^{\alpha}\right)^{a b} V_{\alpha}$ and $V$ of the superalgebra $\operatorname{sl}(1,2)$, with $\left(\sigma^{\alpha}\right)^{a b}$ generating the fundamental representation of $\operatorname{sl}(2)$, may be considered as generators of translations $i P^{a}$, special conformal transformations $i K^{a}$, symplectic rotations $i M^{a b}$ and dilatations $-i D$, respectively, in superspace. This leads immediatly to a 'natural' geometric formulation of the $\operatorname{osp}(1,2)$ quantization procedure: In a superspace description the invariance of $W_{m}$ under $m$-extended BRST transformations, generated by $V_{m}^{a}=V_{+}^{a}+\frac{1}{2} m^{2} V_{-}^{a}$, corresponds to translations combined with $m$-dependent special conformal transformations, and its invariance under $S p(2)$-transformations, generated by $V_{\alpha}$, corresponds to symplectic rotations. Furthermore, solutions $S_{m}$ of the classical master equations $\frac{1}{2}\left(S_{m}, S_{m}\right)^{a}+V_{m}^{a} S_{m}=0$ and $\left\{S_{m}, S_{m}\right\}_{\alpha}+V_{\alpha} S_{m}=0$ with vanishing new ghost number, $\operatorname{ngh}\left(S_{m}\right)=0$, correspond to solutions in the superspace being invariant under dilatations, generated by $V$.

The paper is organized as follows. In Sect. 2 we shortly review some basic definitions and properties of $L$-stage reducible gauge theories and we introduce the corresponding configuration space of fields and antifields. Furthermore, the (anti)commutation relations of the superalgebra $s l(1,2)$ are defined and an explicit realization in terms of linear differential operators acting on the antifields are given. In Sect. 3 the superalgebra $\operatorname{sl}(1,2)$ is realized as algebra of the conformal group in superspace where the usual space-time is extended by two extra anticommuting coordinates $\theta^{a}$. Moreover, we give a superspace representation of the algebra $s l(1,2)$ acting linearly on the super(anti)fields. In Sect. 4 the $\operatorname{osp}(1,2)$-covariant superfield quantization rules for general gauge theories are formulated. Besides, it is shown that proper solutions of the classical master equations can be constructed being invariant under $\operatorname{osp}(1,2) \oplus u(1)$, where the additional $u(1)$ symmetry is related to the new ghost number conservation; however, this symmetry is broken by choosing a gauge. Sect. 5 is devoted to study the (in)dependence of general Green's functions on the choice of the gauge. In the $\operatorname{osp}(1,2)$ approach it is proven that mass terms generally destroy gauge independence; however, this gauge dependence disappears in the limit 
$m=0$. In Sect. 6 we construct $\operatorname{osp}(1,2) \oplus u(1)$ symmetric proper solutions of the classical master equations. Moreover, the problem of how to determine the transformations of the gauge fields and the full set of the necessary (anti)ghost and auxiliary fields under the superalgebra $\operatorname{sl}(1,2)$ is solved both for irreducible and first-stage reducible theories with closed algebra.

Throughout this paper we have used the condensed notation introduced by DeWitt 15 and conventions adopted in Ref. [8]; if not specified otherwise, derivatives with respect to the superantifields $\bar{\Phi}_{A}(\theta)$ and the superspace coordinates $\theta^{a}$ are the (usual) left ones and that with respect to the superfields $\Phi^{A}(\theta)$ are right ones. Left derivatives with respect to $\Phi^{A}(\theta)$ and right derivatives with respect to $\theta^{a}$ are labelled by the subscript $L$ and $R$, respectively; for example, $\delta_{L} / \delta \Phi^{A}(\theta)\left(\partial_{R} / \partial \theta^{a}\right)$ denotes the left(right) derivative with respect to the superfields $\Phi^{A}(\theta)$ (the superspace coordinates $\theta^{a}$ ).

\section{Realization of $\operatorname{sl}(1,2)$ in terms of antifields}

\section{(A) General gauge theories}

Before going into the main subject of this section let us shortly introduce the basic definitions of general gauge theories and the corresponding configuration space of fields and antifields:

A set of gauge (as well as matter) fields $A^{i}$ with Grassmann parities $\epsilon\left(A^{i}\right)=\epsilon_{i}$ will be considered whose classical action $S_{\mathrm{cl}}(A)$ is invariant under the gauge transformations

$$
\delta A^{i}=R_{\alpha_{0}}^{i} \xi^{\alpha_{0}}, \quad \alpha_{0}=1, \ldots, n_{0}, \quad S_{\mathrm{cl}, i} R_{\alpha_{0}}^{i}=0
$$

here, $\xi^{\alpha_{0}}$ are the parameters of these transformations and $R_{\alpha_{0}}^{i}(A)$ are the gauge generators having Grassmann parity $\epsilon\left(\xi^{\alpha_{0}}\right)=\epsilon_{\alpha_{0}}$ and $\epsilon\left(R_{\alpha_{0}}^{i}\right)=\epsilon_{i}+\epsilon_{\alpha_{0}}$, respectively; by definition $X_{, j}=\delta X / \delta A^{j}$.

For general gauge theories the algebra of generators has the form [13]:

$$
R_{\alpha_{0}, j}^{i} R_{\beta_{0}}^{j}-(-1)^{\epsilon_{\alpha_{0}} \epsilon_{\beta_{0}}} R_{\beta_{0}, j}^{i} R_{\alpha_{0}}^{j}=-R_{\gamma_{0}}^{i} F_{\alpha_{0} \beta_{0}}^{\gamma_{0}}-M_{\alpha_{0} \beta_{0}}^{i j} S_{\mathrm{cl}, j},
$$

where $F_{\alpha_{0} \beta_{0}}^{\gamma_{0}}(A)$ are the field-dependent structure functions and the matrix $M_{\alpha_{0} \beta_{0}}^{i j}(A)$ is graded antisymmetric with respect to $(i j)$ and $\left(\alpha_{0} \beta_{0}\right)$. The gauge algebra is said to be closed if $M_{\alpha_{0} \beta_{0}}^{i j}=0$, otherwise it is called open. Moreover, Eq. (2.2) defines a Lie algebra if the algebra is closed and the $F_{\alpha_{0} \beta_{0}}^{\gamma_{0}}$ do not depend on $A^{i}$.

If the set of generators $R_{\alpha_{0}}^{i}$ are linearly independent then the theory is called irreducible [16]. On the other hand, if the generators $R_{\alpha_{0}}^{i}$ are not independent, i.e., if on-shell certain 
relations exist among them, then, according to the following characterization, the theory under consideration is called $L$-stage reducible [17]:

There exists a chain of field--dependent on-shell zero-modes $Z_{\alpha_{s}}^{\alpha_{s}-1}(A)$,

$$
\begin{aligned}
R_{\alpha_{0}}^{i} Z_{\alpha_{1}}^{\alpha_{0}} & =S_{\mathrm{cl}, j} K_{\alpha_{1}}^{j i}, & K_{\alpha_{1}}^{i j} & =-(-1)^{\epsilon_{i} \epsilon_{j}} K_{\alpha_{1}}^{j i}, \\
Z_{\alpha_{s-1}}^{\alpha_{s-2}} Z_{\alpha_{s}}^{\alpha_{s-1}} & =S_{\mathrm{cl}, j} K_{\alpha_{s}}^{j \alpha_{s-2}}, & \alpha_{s} & =1, \ldots, n_{s}, s=2, \ldots, L,
\end{aligned}
$$

where the stage $L$ of reducibility is defined by the lowest value $s$ for which the matrix $Z_{\alpha_{L}}^{\alpha_{L-1}}(A)$ is no longer degenerated. The $Z_{\alpha_{s}}^{\alpha_{s-1}}$ are the on-shell zero modes for $Z_{\alpha_{s-1}}^{\alpha_{s-2}}$ with $\epsilon\left(Z_{\alpha_{s}}^{\alpha_{s-1}}\right)=\epsilon_{\alpha_{s-1}}+\epsilon_{\alpha_{s}}$. In the following, if not stated otherwise, we assume $s$ to take on the values $s=0, \ldots, L$, thereby including also the case of irreducible theories.

The whole space of fields $\phi^{A}$ and antifields $\bar{\phi}_{A}, \phi_{A a}^{*}, \eta_{A}$ together with their Grassmann parities (modulo 2) is characterized by the following sets [13, 8]

$$
\begin{array}{rlrl}
\phi^{A} & =\left(A^{i}, B^{\alpha_{s} \mid a_{1} \cdots a_{s}}, C^{\alpha_{s} \mid a_{0} \cdots a_{s}}, s=0, \ldots L\right), & \epsilon\left(\phi^{A}\right) \equiv \epsilon_{A}=\left(\epsilon_{i}, \epsilon_{\alpha_{s}}+s, \epsilon_{\alpha_{s}}+s+1\right) \\
\bar{\phi}_{A}=\left(\bar{A}_{i}, \bar{B}_{\alpha_{s} \mid a_{1} \cdots a_{s}}, \bar{C}_{\alpha_{s} \mid a_{0} \cdots a_{s}}, s=0, \ldots L\right), & \epsilon\left(\bar{\phi}_{A}\right)=\epsilon_{A}, \\
\phi_{A a}^{*}=\left(A_{i a}^{*}, B_{\alpha_{s} a \mid a_{1} \cdots a_{s}}^{*}, C_{\alpha_{s} a \mid a_{0} \cdots a_{s}}^{*}, s=0, \ldots L\right), & \epsilon\left(\phi_{A a}^{*}\right)=\epsilon_{A}+1, \\
\eta_{A}=\left(D_{i}, E_{\alpha_{s} \mid a_{1} \cdots a_{s}}, F_{\alpha_{s} \mid a_{0} \cdots a_{s}}, s=0, \ldots L\right), & \epsilon\left(\eta_{A}\right)=\epsilon_{A},
\end{array}
$$

respectively. Here, the pyramids of auxiliary fields $B^{\alpha_{s} \mid a_{1} \cdots a_{s}}$ and (anti)ghosts $C^{\alpha_{s} \mid a_{0} \cdots a_{s}}$ are $S p(2)$-tensors of rank $s$ and $s+1$, respectively, being completely symmetric with respect to the 'internal' $S p(2)$-indices $a_{i}=1,2,(i=0,1, \ldots, s)$; similarly for the antifields $\bar{\phi}_{A}, \phi_{A a}^{*}$ and sources $\eta_{A}$. The independent index $a=1,2$ which counts the two components of a $S p(2)$-spinor will be called 'external'. The totally symmetrized $S p(2)$-tensors are irreducible and have maximal $S p(2)$-spin. Raising and lowering of $S p(2)$-indices is obtained by the invariant tensor

$$
\epsilon^{a b}=\left(\begin{array}{cc}
0 & 1 \\
-1 & 0
\end{array}\right), \quad \epsilon^{a c} \epsilon_{c b}=\delta_{b}^{a}
$$

(B) The superalgebra sl(1,2)

The main goal of this Section is to determine the action of the generators of the superalgebra $\operatorname{sl}(1,2)$ on the antifields $\bar{\phi}_{A}, \phi_{A a}^{*}$ and $\eta_{A}$. Let us now introduce that algebra.

The even part of $\operatorname{sl}(1,2) \sim \operatorname{sl}(2,1)$ is the Lie algebra $\operatorname{sl}(2) \oplus u(1)$. We denote by $V_{\alpha},(\alpha=0, \pm)$ the (real) generators of $S L(2)$ and by $V$ the generator of $U(1)$. The odd part of $\operatorname{sl}(1,2)$ contains two (nilpotent) $S L(2)$-spinors, $V_{ \pm}^{a}$, with spin $\frac{1}{2}$ and Weyl weight $\alpha\left(V_{ \pm}^{a}\right)= \pm 1$, respectively. Spin and Weyl weight of $V_{ \pm}^{a}$ are defined through their 
behaviour under the action of the generators $V_{\alpha}$ and $V$, respectively. \&

The (anti)commutation relations of the superalgebra $s l(1,2)$ are [18]:

$$
\begin{aligned}
{\left[V, V_{\alpha}\right] } & =0, & {\left[V, V_{+}^{a}\right] } & =V_{+}^{a}, & {\left[V, V_{-}^{a}\right] } & =-V_{-}^{a}, \\
{\left[V_{\alpha}, V_{\beta}\right] } & =\epsilon_{\alpha \beta}^{\gamma} V_{\gamma}, & {\left[V_{\alpha}, V_{+}^{a}\right] } & =V_{+}^{b}\left(\sigma_{\alpha}\right)_{b}^{a}, & {\left[V_{\alpha}, V_{-}^{a}\right] } & =V_{-}^{b}\left(\sigma_{\alpha}\right)_{b}^{a}, \\
\left\{V_{+}^{a}, V_{+}^{b}\right\} & =0, & \left\{V_{-}^{a}, V_{-}^{b}\right\} & =0, & \left\{V_{+}^{a}, V_{-}^{b}\right\} & =-\left(\sigma^{\alpha}\right)^{a b} V_{\alpha}-\epsilon^{a b} V,
\end{aligned}
$$

where the $S p(2)$-indices are raised or lowered according to

$$
\begin{gathered}
\left(\sigma_{\alpha}\right)^{a b}=\epsilon^{a c}\left(\sigma_{\alpha}\right)_{c}{ }^{b}=\left(\sigma_{\alpha}\right)^{a}{ }_{c} \epsilon^{c b}=\epsilon^{a c}\left(\sigma_{\alpha}\right)_{c d} \epsilon^{d b}, \\
\left(\sigma_{\alpha}\right)_{a}^{b}=-\left(\sigma_{\alpha}\right)^{b}{ }_{a}, \quad\left(\sigma_{\alpha}\right)^{a b}=\left(\sigma_{\alpha}\right)^{b a} .
\end{gathered}
$$

The matrices $\sigma_{\alpha}(\alpha=0, \pm)$ generate the (real) Lie algebra $s l(2)$ being isomorphic to $s p(2)$ :

$$
\begin{gathered}
\left(\sigma_{\alpha}\right)_{a}{ }^{c}\left(\sigma_{\beta}\right)_{c}{ }^{b}=g_{\alpha \beta} \delta_{a}^{b}+\frac{1}{2} \epsilon_{\alpha \beta \gamma}\left(\sigma^{\gamma}\right)_{a}^{b}, \quad\left(\sigma^{\alpha}\right)_{a}^{b}=g^{\alpha \beta}\left(\sigma_{\beta}\right)_{a}^{b} \\
g^{\alpha \beta}=\left(\begin{array}{ccc}
1 & 0 & 0 \\
0 & 0 & 2 \\
0 & 2 & 0
\end{array}\right), \quad g^{\alpha \gamma} g_{\gamma \beta}=\delta_{\beta}^{\alpha},
\end{gathered}
$$

where $\epsilon_{\alpha \beta \gamma}$ is the totally antisymmetric tensor, $\epsilon_{0+-}=1$. For the generators $\sigma_{\alpha}$ we may choose the representation $\left(\sigma_{0}\right)_{a}^{b}=\tau_{3}$ and $\left(\sigma_{ \pm}\right)_{a}^{b}=-\frac{1}{2}\left(\tau_{1} \pm i \tau_{2}\right)$, with $\tau_{\alpha}(\alpha=1,2,3)$ being the Pauli matrices.

Let us now rewrite the $\operatorname{sl}(1,2)$-algebra in two equivalent forms, both of which being of physical relevance in the following. First, introducing another basis $V^{a b}$ of the $S L(2)-$ generators, namely

$$
V^{a b}=\left(\sigma^{\alpha}\right)^{a b} V_{\alpha}
$$

and making use of the equalities

$$
\left(\sigma^{\alpha}\right)^{a b}\left(\sigma_{\alpha}\right)_{d}^{c}=-\epsilon^{c\{a} \delta_{d}^{b\}}, \quad \epsilon_{\alpha \beta}^{\gamma}\left(\sigma^{\alpha}\right)^{a b}\left(\sigma^{\beta}\right)^{c d}=-\epsilon^{\{c\{a}\left(\sigma^{\gamma}\right)^{b\} d\}}
$$

where the curly brackets \{\} indicate symmetrization of indices, the (anti)commutation relations of $\operatorname{sl}(1,2)$ read

$$
\begin{aligned}
& {\left[V, V^{a b}\right]=0,} \\
& {\left[V, V_{+}^{a}\right]=V_{+}^{a},} \\
& {\left[V, V_{-}^{a}\right]=-V_{-}^{a},} \\
& {\left[V^{a b}, V^{c d}\right]=-\epsilon^{\{c\{a} V^{b\} d\}},} \\
& {\left[V^{a b}, V_{+}^{c}\right]=-\epsilon^{c\{a} V_{+}^{b\}},} \\
& {\left[V^{a b}, V_{-}^{c}\right]=-\epsilon^{c\{a} V_{-}^{b\}},} \\
& \left\{V_{+}^{a}, V_{+}^{b}\right\}=0, \\
& \left\{V_{-}^{a}, V_{-}^{b}\right\}=0, \\
& \left\{V_{+}^{a}, V_{-}^{b}\right\}=-V^{a b}-\epsilon^{a b} V \text {. }
\end{aligned}
$$

${ }^{2}$ Identifying $V \equiv i D$ with $D$ being the generator of dilatations in superspace, as will be done in Sect. 3, the Weyl weight coincides with the superspace scale dimension of the corresponding quantity. Of course, the latter should not be confuced with the scale dimension of any quantity in ordinary space-time. 
In that form the superalgebra $s l(1,2)$ may be given a geometric interpretation as the algebra of the conformal group in a 2-dimensional superspace having two anticommuting coordinates (see Sect. 3 below).

Secondly, we remark that within the field-antifield formalism not the entire $s l(1,2)-$ superalgebra will be of physical relevance, since not any of their generators define symmetry operations of the quantum action - only some combinations of them forming a orthosymplectic superalgebra $\operatorname{osp}(1,2)$ generate symmetries (see Sect. 4 below). Therefore, with respect to this let us notice the isomorphism between $\operatorname{sl}(1,2)$ and $\operatorname{osp}(2,2)$ by introducing the following two combinations of $V_{+}^{a}$ and $V_{-}^{a}$,

$$
O_{+}^{a} \equiv V_{+}^{a}+\frac{1}{2} V_{-}^{a}, \quad O_{-}^{a} \equiv V_{+}^{a}-\frac{1}{2} V_{-}^{a} .
$$

Then for the (anti)commutation relations of the superalgebra $\operatorname{osp}(2,2)$ we obtain

$$
\begin{aligned}
& {\left[V, V_{\alpha}\right]=0, \quad\left[V, O_{+}^{a}\right]=O_{-}^{a}, \quad\left[V, O_{-}^{a}\right]=O_{+}^{a},} \\
& {\left[V_{\alpha}, V_{\beta}\right]=\epsilon_{\alpha \beta}^{\gamma} V_{\gamma}, \quad\left[V_{\alpha}, O_{+}^{a}\right]=O_{+}^{b}\left(\sigma_{\alpha}\right)_{b}{ }^{a}, \quad\left[V_{\alpha}, O_{-}^{a}\right]=O_{-}^{b}\left(\sigma_{\alpha}\right)_{b}{ }^{a},} \\
& \left\{O_{+}^{a}, O_{+}^{b}\right\}=-\left(\sigma^{\alpha}\right)^{a b} V_{\alpha}, \quad\left\{O_{-}^{a}, O_{-}^{b}\right\}=\left(\sigma^{\alpha}\right)^{a b} V_{\alpha}, \quad\left\{O_{+}^{a}, O_{-}^{b}\right\}=-\epsilon^{a b} V .
\end{aligned}
$$

Here, $\left(V_{\alpha}, O_{+}^{a}\right)$ as well as $\left(V_{\alpha}, O_{-}^{a}\right)$ obey two different $\operatorname{osp}(1,2)$-superalgebras with $\left(V, O_{-}^{a}\right)$ as well as $\left(V, O_{+}^{a}\right)$ forming an irreducible tensor of these algebras, respectively, either of them transforming according to the same representation. Notice, that both $O_{+}^{a}$ and $O_{-}^{a}$ are neither nilpotent nor do they anticommute among themselves.

(C) Representation of $\operatorname{sl}(1,2)$ on the antifields

Now, let us give an explicit linear realization of the generators of the superalgebra (2.3) by their action on the antifields $\bar{\phi}_{A}, \phi_{A a}^{*}$ and the sources $\eta_{A}$ (a nonlinear realization on the fields $\phi^{A}$ will be given in Sect. 4).

$$
\begin{array}{rlrl}
V_{+}^{a} \bar{\phi}_{A} & =\epsilon^{a b} \phi_{A b}^{*}, & V_{-}^{a} \bar{\phi}_{A} & =0, \\
V_{+}^{a} \phi_{A b}^{*} & =-\delta_{b}^{a} \eta_{A}, & V_{-}^{a} \phi_{A b}^{*} & =\bar{\phi}_{B}\left(\left(\sigma^{\alpha}\right)_{b}^{a}\left(\sigma_{\alpha}\right)_{A}^{B}-\delta_{b}^{a} \bar{\gamma}_{A}^{B}\right), \\
V_{+}^{a} \eta_{A} & =0, & V_{-}^{a} \eta_{A} & =\phi_{B b}^{*}\left(\left(\sigma^{\alpha}\right)^{a b}\left(\sigma_{\alpha}\right)^{B}{ }_{A}-\epsilon^{a b}\left(\bar{\gamma}_{A}^{B}+2 \delta_{A}^{B}\right)\right), \\
V_{\alpha} \bar{\phi}_{A} & =\bar{\phi}_{B}\left(\sigma_{\alpha}\right)_{A}^{B}, \\
V_{\alpha} \phi_{A b}^{*} & =\phi_{B b}^{*}\left(\sigma_{\alpha}\right)_{A}^{B}+\phi_{A a}^{*}\left(\sigma_{\alpha}\right)_{b}^{a}, & V \bar{\phi}_{A} & =\bar{\phi}_{B} \bar{\gamma}_{A}^{B}, \\
V_{\alpha} \eta_{A} & =\eta_{B}\left(\sigma_{\alpha}\right)_{A}^{B}{ }_{A}, & & =\phi_{B b}^{*}\left(\bar{\gamma}_{A}^{B}+\delta_{A}^{B}\right), \\
V \eta_{A} & =\eta_{B}\left(\bar{\gamma}_{A}^{B}+2 \delta_{A}^{B}\right)
\end{array}
$$

(for a componentwise notation see Appendix A). In Eqs. (2.7), (2.8) we introduced two kinds of matrices which deserve some explanation. The matrices $\left(\sigma_{\alpha}\right)^{B}{ }_{A}$ are generalized 
$\sigma$-matrices acting only on internal $S p(2)$-indices of the (anti)fields, for example,

$$
\bar{\phi}_{B}\left(\sigma_{\alpha}\right)^{B}{ }_{A}=\left(0, \sum_{r=1}^{s} \bar{B}_{\alpha_{s} \mid a_{1} \cdots a_{r-1} a a_{r+1} \cdots a_{s}}\left(\sigma_{\alpha}\right)^{a} a_{r}, \sum_{r=0}^{s} \bar{C}_{\alpha_{s} \mid a_{0} \cdots a_{r-1} a a_{r+1} \cdots a_{s}}\left(\sigma_{\alpha}\right)^{a} a_{r}\right)
$$

their general definition is given by

$$
\left(\sigma_{\alpha}\right)_{A}^{B} \equiv \begin{cases}\delta_{\alpha_{s}}^{\beta_{s}}(s+1)\left(\sigma_{\alpha}\right)^{b}{ }_{a} S_{a_{1} \cdots b_{s} b}^{b_{1} \cdots b_{s} a} & \text { for } A=\alpha_{s}\left|a_{1} \cdots a_{s}, B=\beta_{s}\right| b_{1} \cdots b_{s} \\ \delta_{\alpha_{s}}^{\beta_{s}}(s+2)\left(\sigma_{\alpha}\right)^{b}{ }_{a} S_{a_{0} \cdots a_{s} b} S_{0} \cdots b_{s} a & \text { for } A=\alpha_{s}\left|a_{0} \cdots a_{s}, B=\beta_{s}\right| b_{0} \cdots b_{s} \\ 0 & \text { otherwise }\end{cases}
$$

where the symmetrizer $S_{a_{0} \cdots a_{s} b}^{b_{0} \cdots b_{s} a}$ is defined as

$$
S_{a_{0} \cdots a_{s} b}^{b_{0} \cdots b_{s} a} \equiv \frac{1}{(s+2) !} \frac{\partial}{\partial X^{a_{0}}} \cdots \frac{\partial}{\partial X^{a_{s}}} \frac{\partial}{\partial X^{b}} X^{a} X^{b_{s}} \cdots X^{b_{0}}
$$

$X^{a}$ being independent bosonic variables. These operators, obeying $S_{c_{0} \cdots c_{s} d}^{b_{0} \cdots b_{s} a} S_{a_{0} \cdots a_{s} b}^{c_{0} \cdots c_{s} d}=$ $S_{a_{0} \cdots a_{s} b}^{b_{0} \cdots b_{s} a}$, possess the additional properties

$$
\begin{aligned}
S_{a_{0} \cdots a_{s} b}^{b_{0} \cdots b_{s} a} & =\frac{1}{s+2}\left(\sum_{r=0}^{s} \delta_{a_{0}}^{b_{r}} S_{a_{1} \cdots a_{s} b}^{b_{0} \cdots b_{r-1} b_{r+1} \cdots b_{s} a}+\frac{1}{s+1} \sum_{r=0}^{s} \delta_{a_{0}}^{a} \delta_{b}^{b_{r}} S_{a_{1} \cdots a_{s}}^{b_{0} \cdots b_{r-1} b_{r+1} \cdots b_{s}}\right), \\
S_{a_{0} \cdots a_{s}}^{b_{0} \cdots b_{s}} & =\frac{1}{s+1} \sum_{r=0}^{s} \delta_{a_{0}}^{b_{r}} S_{a_{1} \cdots a_{s}}^{b_{0} \cdots b_{r-1} b_{r+1} \cdots b_{s}} .
\end{aligned}
$$

Furthermore, $\bar{\gamma}_{A}^{B}=\alpha\left(\bar{\phi}_{A}\right) \delta_{A}^{B}$ are arbitrary diagonal matrices whose entries $\alpha\left(\bar{\phi}_{A}\right)$, in general, may be any (real) numbers. By definition, cf. Eq. (2.8), $\alpha\left(\bar{\phi}_{A}\right)$ is the (up to now arbitrary) Weyl weight of the antifields $\bar{\phi}$. (This arbitrariness may be traced back to fact that these representations of $\operatorname{sl}(1,2)$ are not completely reducible, cf. [18]). Taking advantage of that freedom we may fix $\alpha\left(\bar{\phi}_{A}\right)$ by relating it to the Weyl weight $\alpha\left(\phi^{A}\right)$ of the fields $\phi^{A}$ - which is uniquely determined by means of the quantum master equations at the lowest order of $\hbar$ (see Sect. 4 and 6 below) - according to

$$
\bar{\gamma}_{A}^{B}+\gamma_{A}^{B}+2 \delta_{A}^{B}=0, \quad \text { i.e., } \quad \alpha\left(\bar{\phi}_{A}\right)+\alpha\left(\phi^{A}\right)+2=0,
$$

where $\gamma_{A}^{B}=\alpha\left(\phi^{A}\right) \delta_{A}^{B}$ is the analogous (diagonal) matrix in the $s l(1,2)$-representations of the fields [? These matrices $\gamma_{A}^{B}$ are given by

$$
\gamma_{A}^{B} \equiv \begin{cases}\delta_{\alpha_{s}}^{\beta_{s}}(s+2) \delta_{a_{1}}^{b_{1}} \cdots \delta_{a_{s}}^{b_{s}} & \text { for } A=\alpha_{s}\left|a_{1} \cdots a_{s}, B=\beta_{s}\right| b_{1} \cdots b_{s}, \\ \delta_{\alpha_{s}}^{\beta_{s}}(s+1) \delta_{a_{0}}^{b_{0}} \cdots \delta_{a_{s}}^{b_{s}} & \text { for } A=\alpha_{s}\left|a_{0} \cdots a_{s}, B=\beta_{s}\right| b_{0} \cdots b_{s}, \\ 0 & \text { otherwise }\end{cases}
$$

3 The requirement (2.10) ensures that (proper) solutions $S_{m}$ of the $m$-extended classical master equations can be constructed having vanishing Weyl weight, $\alpha\left(S_{m}\right)=0$. Later on, we identify the Weyl weight of the (anti)fields with the new ghost number introduced in Ref. [13]. 
From their entries one may read off the Weyl weight $\alpha\left(\phi^{A}\right)$ of the fields $\phi^{A}$, namely

$$
\alpha\left(\phi^{A}\right)=(0, s+2, s+1),
$$

and, throught Eq. (2.10), the Weyl weights of the antifields $\bar{\phi}_{A}, \phi_{A a}^{*}$ and $\eta_{A}$,

$$
\alpha\left(\bar{\phi}_{A}\right)=-\alpha\left(\phi^{A}\right)-2, \quad \alpha\left(\phi_{A a}^{*}\right)=-\alpha\left(\phi^{A}\right)-1, \quad \alpha\left(\eta_{A}\right)=-\alpha\left(\phi^{A}\right) .
$$

In order to prove that the transformations (2.7) and (2.8) obey the $\operatorname{sl}(1,2)$-superalgebra one needs the basic properties (2.4) of the matrices $\sigma_{\alpha}$ and the following two equalities:

$$
\begin{aligned}
\epsilon^{a c} \delta_{d}^{b}+\epsilon^{b c} \delta_{d}^{a} & =-\left(\sigma^{\alpha}\right)^{a b}\left(\sigma_{\alpha}\right)_{d}^{c}, \\
\left(\sigma^{\alpha}\right)^{a b}\left(\left(\sigma_{\alpha}\right)^{c} e_{e_{f}}^{d}+\delta_{e}^{c}\left(\sigma_{\alpha}\right)_{f}^{d}\right) & =\left(\sigma^{\alpha}\right)^{a b}\left(\left(\sigma_{\alpha}\right)^{d} e_{f}^{c}+\delta_{e}^{d}\left(\sigma_{\alpha}\right)_{f}^{c}\right),
\end{aligned}
$$

which can be proven by means of the following relations:

$$
\epsilon^{a b} \delta_{d}^{c}+\epsilon^{b c} \delta_{d}^{a}+\epsilon^{c a} \delta_{d}^{b}=0, \quad \epsilon^{a b}\left(\delta_{e}^{c} \delta_{f}^{d}-\delta_{e}^{d} \delta_{f}^{c}\right)=\epsilon^{c d}\left(\delta_{e}^{a} \delta_{f}^{b}-\delta_{e}^{b} \delta_{f}^{a}\right) .
$$

\section{Superspace representations of the algebra $\operatorname{sl}(1,2)$}

This Section is devoted to a geometric interpretation of the superalgebra $\operatorname{sl}(1,2)$ as given by Eqs. (2.6). This opens the possibility to formulate the quantization of general gauge theories in terms of super(anti)fields over a 2-dimensional superspace.

(A) Representations of $\operatorname{sl}(1,2)$ in superspace

In Ref. [14 it was pointed out that the generators of the (real) algebra $\operatorname{osp}(1,1 \mid 2) \sim$ $s l(1,2)$ acquire a clear geometric meaning if they are interpreted as generators of transformations in superspace. This is obtained by redefining the generators of $\operatorname{sl}(1,2)$ as follows:

$$
V_{+}^{a} \equiv-i P^{a}, \quad V_{-}^{a} \equiv-i K^{a}, \quad V^{a b} \equiv-i M^{a b}, \quad V \equiv i D
$$

Then, the (anti)commutation relations resulting from (2.6) can be interpreted as algebra of the conformal group in two anticommuting dimensions with metric tensor $\epsilon^{a b}$ :

$$
\begin{aligned}
& {\left[D, M^{a b}\right]=0, \quad\left[D, P^{a}\right]=-i P^{a}, \quad\left[D, K^{a}\right]=i K^{a},} \\
& {\left[M^{a b}, M^{c d}\right]=-i \epsilon^{\{c\{a} M^{b\} d\}}, \quad\left[M^{a b}, P^{c}\right]=-i \epsilon^{c\{a} P^{b\}}, \quad\left[M^{a b}, K^{c}\right]=-i \epsilon^{c\{a} K^{b\}},} \\
& \left\{P^{a}, P^{b}\right\}=0, \quad\left\{K^{a}, K^{b}\right\}=0, \quad\left\{P^{a}, K^{b}\right\}=i\left(\epsilon^{a b} D-M^{a b}\right),
\end{aligned}
$$

with $P^{a}, K^{a}, M^{a b}$ and $D$ being the generators of translations, special conformal transformations, (symplectic) rotations and dilatations, respectively. The superspace which we 
encounter here is obtained by extending the usual spacetime to include two extra anticommuting coordinates $\theta^{a}$. Raising and lowering of $S p(2)$-indices are defined by the rules $\theta^{a}=\epsilon^{a b} \theta_{b}$ and $\theta_{a}=\epsilon_{a b} \theta^{b}$; the square of $\theta^{a}$ and the derivative with respect to it are defined by $\theta^{2} \equiv \frac{1}{2} \epsilon_{a b} \theta^{b} \theta^{a}$ and $\partial^{2} / \partial \theta^{2} \equiv \frac{1}{2} \epsilon^{a b} \partial^{2} / \partial \theta^{b} \partial \theta^{a}$.

The representation of the algebra (3.2) in that superspace is given by

$$
\begin{aligned}
P^{a} & =i \frac{\partial}{\partial \theta_{a}}, \\
K^{a} & =2 i \theta^{2} \frac{\partial}{\partial \theta_{a}}-\theta_{b}\left(\Sigma^{a b}-i \epsilon^{a b} \Delta\right), \\
M^{a b} & =-i\left(\theta^{a} \frac{\partial}{\partial \theta_{b}}+\theta^{b} \frac{\partial}{\partial \theta_{a}}\right)+\Sigma^{a b}, \\
D & =i \theta_{a} \frac{\partial}{\partial \theta_{a}}-i \Delta,
\end{aligned}
$$

where $\Sigma^{a b}$ and $\Delta$ constitute the basis of some finite-dimensional representation of the algebra of the "little group", i.e., the stabilizer subgroup of that conformal group,

$$
\left[\Sigma^{a b}, \Sigma^{c d}\right]=-\epsilon^{\{c\{a} \Sigma^{b\} d\}}, \quad\left[\Delta, \Sigma^{a b}\right]=0 .
$$

Obviously, the corresponding representation of the algebra (2.6) is obtained by a change of the $S L(2)$-generators analogous to 2.5$), \Sigma^{a b}=i\left(\sigma^{\alpha}\right)^{a b} \Sigma_{\alpha}$, with $\Sigma_{\alpha}$ being related to the matrix representation of the $V_{\alpha}$ 's and satisfying

$$
\left[\Sigma_{\alpha}, \Sigma_{\beta}\right]=\epsilon_{\alpha \beta}^{\gamma} \Sigma_{\gamma}, \quad\left[\Delta, \Sigma_{\alpha}\right]=0
$$

The corresponding representation of the generators (3.1) in the superspace are

$$
\begin{aligned}
V_{+}^{a} & =\frac{\partial}{\partial \theta_{a}} \\
V_{-}^{a} & =2 \theta^{2} \frac{\partial}{\partial \theta_{a}}-\theta_{b}\left(\left(\sigma^{\alpha}\right)^{a b} \Sigma_{\alpha}-\epsilon^{a b} \Delta\right), \\
V_{\alpha} & =\theta^{a}\left(\sigma_{\alpha}\right)^{a}{ }_{b} \frac{\partial}{\partial \theta_{b}}+\Sigma_{\alpha}, \\
V & =-\theta_{a} \frac{\partial}{\partial \theta_{a}}+\Delta .
\end{aligned}
$$

(B) Representation of $\operatorname{sl}(1,2)$ on super(anti)fields

Now, having revealed the geometrical content of the generators of $\operatorname{sl}(1,2)$ we are able to formulate the transformations (2.7) and (2.8) in superspace. Let $\Phi^{A}(\theta), \epsilon\left(\Phi^{A}(\theta)\right) \equiv \epsilon_{A}$, be a set of superfields with the restriction $\left.\Phi^{A}(\theta)\right|_{\theta=0}=\phi^{A}$. It admits the following general expansion in terms of component fields,

$$
\Phi^{A}(\theta)=\phi^{A}+\pi^{A a} \theta_{a}-\lambda^{A} \theta^{2}, \quad \frac{\delta}{\delta \Phi^{A}(\theta)}=\frac{\delta}{\delta \phi^{A}} \theta^{2}-\theta^{a} \frac{\delta}{\delta \pi^{A a}}-\frac{\delta}{\delta \lambda^{A}}
$$


(remember that, according to the general convention, derivatives with respect to the fields are defined as acting from the right). With each superfield $\Phi^{A}(\theta)$ a superantifield $\bar{\Phi}_{A}(\theta)$ is associated having the same Grassmann parity, $\epsilon\left(\bar{\Phi}_{A}(\theta)\right)=\epsilon_{A}$,

$$
\bar{\Phi}_{A}(\theta)=\bar{\phi}_{A}-\theta^{a} \phi_{A a}^{*}-\theta^{2} \eta_{A}, \quad \frac{\delta}{\delta \bar{\Phi}_{A}(\theta)}=\theta^{2} \frac{\delta}{\delta \bar{\phi}_{A}}+\frac{\delta}{\delta \phi_{A a}^{*}} \theta_{a}-\frac{\delta}{\delta \eta_{A}} .
$$

According to (3.12) and (3.13) for the expressions of the derivatives it holds

$$
\frac{\delta \Phi^{A}(\theta)}{\delta \Phi^{B}(\bar{\theta})}=\frac{\delta \bar{\Phi}_{B}(\theta)}{\delta \bar{\Phi}_{A}(\bar{\theta})}=\delta_{B}^{A} \delta^{2}(\theta-\bar{\theta}), \quad \text { with } \quad \delta^{2}(\theta-\bar{\theta}) \equiv(\theta-\bar{\theta})^{2} .
$$

Then, by the help of $\bar{\Phi}_{A}(\theta)$ the $\operatorname{sl}(1,2)$-transformations (2.7) and (2.8) may be written in the following compact form:

$$
\begin{aligned}
V_{+}^{a} \bar{\Phi}_{A}(\theta) & =\frac{\partial \bar{\Phi}_{A}(\theta)}{\partial \theta_{a}} \\
V_{-}^{a} \bar{\Phi}_{A}(\theta) & =2 \theta^{2} \frac{\partial \bar{\Phi}_{A}(\theta)}{\partial \theta_{a}}-\theta_{b}\left(\left(\sigma^{\alpha}\right)^{a b} \Sigma_{\alpha}-\epsilon^{a b} \Delta\right) \bar{\Phi}_{A}(\theta) \\
V_{\alpha} \bar{\Phi}_{A}(\theta) & =-\left\{\theta_{a}\left(\sigma_{\alpha}\right)_{b}^{a} \frac{\partial \bar{\Phi}_{A}(\theta)}{\partial \theta_{b}}+\Sigma_{\alpha} \bar{\Phi}_{A}(\theta)\right\} \\
V \bar{\Phi}_{A}(\theta) & =-\left\{-\theta_{a} \frac{\partial \bar{\Phi}_{A}(\theta)}{\partial \theta_{a}}+\Delta \bar{\Phi}_{A}(\theta)\right\}
\end{aligned}
$$

with

$$
\Sigma_{\alpha} \bar{\Phi}_{A}(\theta)=-\bar{\Phi}_{B}(\theta)\left(\sigma_{\alpha}\right)_{A}^{B}, \quad \Delta \bar{\Phi}_{A}(\theta)=-\bar{\Phi}_{B}(\theta) \bar{\gamma}_{A}^{B}
$$

Some care has to be taken in order to get the correct signs in these equations. First, in order to attain that the transformations laws (3.14)-(3.17) are compatible with the superalgebra (2.6) it is necessary to take into account an extra minus sign on the righthand side of (3.16) and (3.17) (cf. Eqs. (3.10), (3.11)). Since the matrices $\Sigma_{\alpha}$ generate an irreducible representation of the symplectic group, by virtue of (3.7), $-\Delta$ must be a number which, by definition, agrees with the Weyl weight of the superantifields (observe $\alpha(\theta)=1$ in accordance with Eqs. (2.13)). Secondly, let us emphasize that the minus sign on the right-hand side of the first relation (3.18) is crucial: A further transformation in (3.16) does not act on the numerical matrices $\Sigma_{\alpha}$ but directly on $\bar{\Phi}_{A}(\theta)$; this reverses the factors on the right-hand side against those on the left one and the minus sign is therefore necessary to retain the multiplication law of the conformal group.

Collecting the results obtained up to now the representation of the generators of $\operatorname{sl}(1,2)$ 
by differential operators on the superspace reads

$$
\begin{aligned}
V_{+}^{a} & =\int d^{2} \theta \frac{\partial \bar{\Phi}_{A}(\theta)}{\partial \theta_{a}} \frac{\delta}{\delta \bar{\Phi}_{A}(\theta)} \\
V_{-}^{a} & =\int d^{2} \theta\left\{2 \theta^{2} \frac{\partial \bar{\Phi}_{A}(\theta)}{\partial \theta_{a}}+\theta_{b} \bar{\Phi}_{B}(\theta)\left(\left(\sigma^{\alpha}\right)^{a b}\left(\sigma_{\alpha}\right)^{B}{ }_{A}-\epsilon^{a b} \bar{\gamma}_{A}^{B}\right)\right\} \frac{\delta}{\delta \bar{\Phi}_{A}(\theta)} \\
V_{\alpha} & =\int d^{2} \theta\left\{-\theta_{a}\left(\sigma_{\alpha}\right)_{b}{ }_{b} \frac{\partial \bar{\Phi}_{A}(\theta)}{\partial \theta_{b}}+\bar{\Phi}_{B}(\theta)\left(\sigma_{\alpha}\right)^{B}{ }_{A}\right\} \frac{\delta}{\delta \bar{\Phi}_{A}(\theta)} \\
V & =\int d^{2} \theta\left\{\theta_{a} \frac{\partial \bar{\Phi}_{A}(\theta)}{\partial \theta_{a}}+\bar{\Phi}_{B}(\theta) \bar{\gamma}_{A}^{B}\right\} \frac{\delta}{\delta \bar{\Phi}_{A}(\theta)}
\end{aligned}
$$

where the integration over $\theta^{a}$ is given by

$$
\int d^{2} \theta=0, \quad \int d^{2} \theta \theta^{a}=0, \quad \int d^{2} \theta \theta^{a} \theta^{b}=\epsilon^{a b}
$$

Making use of the expansions (3.13) for $\bar{\Phi}_{A}(\theta)$ and $\delta / \delta \bar{\Phi}_{A}(\theta)$ and performing in Eqs. (3.19)-(3.22) the $\theta$-integration it is easily verified that the resulting expressions for $V_{ \pm}^{a}$, $V_{\alpha}$ and $V$ generate exactly the transformations (2.7) and (2.8) of the component fields of $\bar{\Phi}_{A}(\theta)$.

Furthermore, let us give also a superspace representation of $\operatorname{sl}(1,2)$ in terms of $\Phi^{A}(\theta)$. The corresponding generators $U_{ \pm}^{a}, U_{\alpha}$ and $U$ being defined as right derivatives - in contrast to $V_{ \pm}^{a}, V_{\alpha}$ and $V$, which are defined as left ones - obey the following (anti)commutation relations (cf. Eqs. (2.3))

$$
\begin{aligned}
{\left[U, U_{\alpha}\right] } & =0, & {\left[U, U_{+}^{a}\right] } & =-U_{+}^{a}, & {\left[U, U_{-}^{a}\right] } & =U_{-}^{a}, \\
{\left[U_{\alpha}, U_{\beta}\right] } & =-\epsilon_{\alpha \beta}^{\gamma} U_{\gamma}, & {\left[U_{\alpha}, U_{+}^{a}\right] } & =-U_{+}^{b}\left(\sigma_{\alpha}\right)_{b}{ }^{a}, & {\left[U_{\alpha}, U_{-}^{a}\right] } & =-U_{-}^{b}\left(\sigma_{\alpha}\right)_{b}{ }^{a}, \\
\left\{U_{+}^{a}, U_{+}^{b}\right\} & =0, & \left\{U_{-}^{a}, U_{-}^{b}\right\} & =0, & \left\{U_{+}^{a}, U_{-}^{b}\right\} & =\left(\sigma^{\alpha}\right)^{a b} U_{\alpha}+\epsilon^{a b} U .
\end{aligned}
$$

If we replace in Eqs. (3.19)-(3.22) the superantifield $\bar{\Phi}_{A}(\theta)$ by $\Phi^{A}(\theta)$, the left derivatives $\delta_{L} / \delta \bar{\Phi}_{A}(\theta)$ by the right derivatives $\delta_{R} / \delta \Phi^{A}(\theta)$, and reverse the order of all the factors, then for the representations we are looking for we obtain

$$
\begin{aligned}
U_{+}^{a} & =\int d^{2} \theta \frac{\delta}{\delta \Phi^{A}(\theta)} \frac{\partial_{R} \Phi^{A}(\theta)}{\partial \theta_{a}} \\
U_{-}^{a} & =\int d^{2} \theta \frac{\delta}{\delta \Phi^{A}(\theta)}\left\{2 \theta^{2} \frac{\partial_{R} \Phi^{A}(\theta)}{\partial \theta_{a}}+\left(\left(\sigma^{\alpha}\right)^{a b}\left(\sigma_{\alpha}\right)_{B}^{A}+\epsilon^{a b} \gamma_{B}^{A}\right) \Phi^{B}(\theta) \theta_{b}\right\} \\
U_{\alpha} & =\int d^{2} \theta \frac{\delta}{\delta \Phi^{A}(\theta)}\left\{-\frac{\partial_{R} \Phi^{A}(\theta)}{\partial \theta_{b}}\left(\sigma_{\alpha}\right)_{b}{ }^{a} \theta_{a}+\left(\sigma_{\alpha}\right)^{A}{ }_{B} \Phi^{B}(\theta)\right\} \\
U & =\int d^{2} \theta \frac{\delta}{\delta \Phi^{A}(\theta)}\left\{\frac{\partial_{R} \Phi^{A}(\theta)}{\partial \theta_{a}} \theta_{a}+\gamma_{B}^{A} \Phi^{B}(\theta)\right\} .
\end{aligned}
$$


In addition, we have replaced $\bar{\gamma}_{A}^{B}$ by the (diagonal) matrix $\gamma_{A}^{B}=\alpha\left(\phi^{A}\right) \delta_{A}^{B}$, whose entries $\alpha\left(\phi^{A}\right)$ are given by Eq. (2.12).

Making use of the expansions (3.13) for $\Phi^{A}(\theta)$ and $\delta / \delta \Phi^{A}(\theta)$ and integrating in Eqs. (3.24)-(3.27) over $\theta^{a}$ for the components of $\Phi^{A}(\theta)$ one obtains the (linear) transformations

$$
\begin{aligned}
\phi^{A} U_{+}^{a} & =\pi^{A a}, & \phi^{A} U_{-}^{a} & =0 \\
\pi^{A b} U_{+}^{a} & =-\epsilon^{a b} \lambda^{A}, & \pi^{A b} U_{-}^{a} & =\left(\left(\sigma^{\alpha}\right)^{a b}\left(\sigma_{\alpha}\right)^{A}{ }_{B}+\epsilon^{a b} \gamma_{B}^{A}\right) \phi^{B} \\
\lambda^{A} U_{+}^{a} & =0, & \lambda^{A} U_{-}^{a} & =\left(\left(\sigma^{\alpha}\right)_{b}^{a}\left(\sigma_{\alpha}\right)^{A}{ }_{B}+\delta_{b}^{a}\left(\gamma_{B}^{A}+2 \delta_{B}^{A}\right)\right) \pi^{B b} \\
\phi^{A} U_{\alpha} & =\left(\sigma_{\alpha}\right)^{A}{ }_{B} \phi^{B}, & \phi^{A} U & =\gamma_{B}^{A} \phi^{B}, \\
\pi^{A a} U_{\alpha} & =\left(\sigma_{\alpha}\right)^{A}{ }_{B} \pi^{B a}+\left(\sigma_{\alpha}\right)^{a}{ }_{b} \pi^{A b}, & \pi^{A a} U & =\left(\gamma_{B}^{A}+\delta_{B}^{A}\right) \pi^{B a}, \\
\lambda^{A} U_{\alpha} & =\left(\sigma_{\alpha}\right)^{A}{ }_{B} \lambda^{B}, & \lambda^{A} U & =\left(\gamma_{B}^{A}+2 \delta_{B}^{A}\right) \lambda^{B},
\end{aligned}
$$

which define the explicit realization of $s l(1,2)$ on the superfield analogous to Eqs. (2.7) and (2.8). By a simple straightforward calculation it is verified that the transformations (3.28) and (3.29) indeed satisfy the $s l(1,2)$-superalgebra (3.23).

\section{Quantum master equations}

The superspace representation of $s l(1,2)$ obtained in the previous section enables one to attack the problem of superfield quantization of general gauge theories. A superfield version for the $S p(2)$-covariant Lagrangian quantization was proposed in Ref. [6]. In that approach the quantum action $W\left(\Phi^{A}(\theta), \bar{\Phi}_{A}(\theta)\right)$ is required to be invariant under the (anti)BRST transformations which, in superspace, are realized as translations along the coordinates $\theta^{a}$.

In order to proceed further in the development of that formalism one may attempt to include also special conformal transformations, symplectic rotations and dilatations by imposing additional symmetry requirements. Such an extension is possible, but only for one of the two $\operatorname{osp}(1,2)$-subalgebras of $\operatorname{osp}(2,2) \sim \operatorname{sl}(1,2)$. Indeed, for a superfield description of the $\operatorname{osp}(1,2)$-covariant quantization procedure introduced in Ref. [8] one needs both translations as well as special conformal transformations and symplectic rotations. In that approach the translations are combined with the special conformal transformations by means of a mass parameter $m$ leading to $m$-dependent (anti)BRST transformations. The invariance under symplectic transformations ensures the ghost number conservation of the corresponding quantum action $W_{m}\left(\Phi^{A}(\theta), \bar{\Phi}_{A}(\theta)\right)$. In addition, the dilatations may 
be used to ensure the new ghost number conservation of $W_{m}\left(\Phi^{A}(\theta), \bar{\Phi}_{A}(\theta)\right)$ at the lowest order of $\hbar$.

\section{(A) Sp(2)-covariant superfield quantization}

To begin with, we shortly review the $S p(2)$-covariant superfield quantization [6]. Let us introduce the antisymplectic differential operators

$$
\bar{\Delta}^{a}=\Delta^{a}+(i / \hbar) V^{a}, \quad V^{a} \equiv V_{+}^{a},
$$

with the translations $V_{+}^{a}$ given by Eq. (3.19) and the nilpotent (second-order) differential operators $\Delta^{a}$ given by

$$
\Delta^{a}=\int d^{2} \theta \frac{\partial^{2} \delta_{L}}{\partial \theta^{2} \delta \Phi^{A}(\theta)} \theta^{a} \frac{\delta}{\delta \bar{\Phi}_{A}(\theta)}=(-1)^{\epsilon_{A}} \frac{\delta_{L}}{\delta \phi^{A}} \frac{\delta}{\delta \phi_{A a}^{*}} .
$$

Let us remark, that this definition of $\Delta^{a}$ by projecting out from $\delta_{L} / \delta \Phi^{A}(\theta)$ only the first component agrees with the initial definition in Ref. [13] but differs from that in Ref. [6]. In our opinion the definition (4.2) seems to be much better adapted to the present aim than that of Ref. [6] since a change of the definition of $\Delta^{a}$, like in the triplectic quantization [19], requires also a change of the definition of $V^{a}$ - but then the geometric meaning of $V^{a}$ would be lost. The operators $\bar{\Delta}^{a}, \Delta^{a}$ and $V^{a}$ possess the important properties of nilpotency and (relative) anticommutativity,

$$
\left\{\bar{\Delta}^{a}, \bar{\Delta}^{b}\right\}=0 \quad \Longleftrightarrow \quad\left\{\Delta^{a}, \Delta^{b}\right\}=0, \quad\left\{V^{a}, V^{b}\right\}=0, \quad\left\{\Delta^{a}, V^{b}\right\}=0 .
$$

The basic object of the superfield quantization is the quantum action $W\left(\Phi^{A}(\theta), \bar{\Phi}_{A}(\theta)\right)$, which is required to be a solution of the quantum master equation

$$
\bar{\Delta}^{a} \exp \{(i / \hbar) W\}=0 \quad \Longleftrightarrow \quad \frac{1}{2}(W, W)^{a}+V^{a} W=i \hbar \Delta^{a} W
$$

where the superantibrackets $(F, G)^{a}$ are defined by

$$
(F, G)^{a}=(-1)^{\epsilon_{A}} \int d^{2} \theta\left\{\frac{\partial^{2} \delta F}{\partial \theta^{2} \delta \Phi^{A}(\theta)} \theta^{a} \frac{\delta G}{\delta \bar{\Phi}_{A}(\theta)}-(-1)^{(\epsilon(F)+1)(\epsilon(G)+1)}(F \leftrightarrow G)\right\} .
$$

The solution of (4.3) is sought of as a power series in Planck's constant $\hbar$,

$$
W=S+\sum_{n=1}^{\infty} \hbar^{n} W_{n}
$$

Furthermore, two requirements - the nondegeneracy of $S$ and the correctness of the classical limit - have to be imposed. The first one is translated into the requirement that $S$ should be a proper solution of the classical master equation, i.e., the Hessian of second 
derivatives of $S$ should be of maximal rank at the stationary points, and the second one means that $S$ should satisfy the usual boundary condition, namely that $S$ coincides with the classical action $S_{\mathrm{cl}}(A)$ if all the antifields are put equal to zero.

To remove the gauge degeneracy of the action $S$, one introduces the operator

$$
\hat{U}(F)=\exp \{(\hbar / i) \hat{T}(F)\} \quad \text { with } \quad \hat{T}(F)=\frac{1}{2} \epsilon_{a b}\left\{\bar{\Delta}^{b},\left[\bar{\Delta}^{a}, F\right]\right\}
$$

$F=F\left(\Phi^{A}(\theta)\right)$ being an arbitrary bosonic gauge fixing functional. Then, the gauge fixed quantum action $W_{\text {ext }}\left(\Phi^{A}(\theta), \bar{\Phi}_{A}(\theta)\right)$, defined by

$$
\exp \left\{(i / \hbar) W_{\text {ext }}\right\}=\hat{U}(F) \exp \{(i / \hbar) W\}
$$

is also a solution of the quantum master equations (4.3).

(B) osp (1,2)-covariant superfield quantization

Let us now give the superfield description of the $\operatorname{osp}(1,2)$-covariant quantization [8]. In that approach the antisymplectic differential operators (4.1) are replaced by

$$
\bar{\Delta}_{m}^{a}=\Delta^{a}+(i / \hbar) V_{m}^{a}, \quad V_{m}^{a} \equiv V_{+}^{a}+\frac{1}{2} m^{2} V_{-}^{a}
$$

with the special conformal operators $V_{-}^{a}$ given by Eq. (3.20). Here, the mass parameter $m$ having Weyl weight $\alpha(m)=1$ is introduced because $V_{+}^{a}$ and $V_{-}^{a}$ have different mass dimensions (and opposite Weyl weight $\alpha\left(V_{ \pm}^{a}\right)= \pm 1$ ). In addition, one introduces the differential operators

$$
\bar{\Delta}_{\alpha}=\Delta_{\alpha}+(i / \hbar) V_{\alpha}
$$

with the symplectic rotations $V_{\alpha}$ given by Eq. (3.21) and the (second-order) differential operators $\Delta_{\alpha}$ being defined by

$$
\Delta_{\alpha}=(-1)^{\epsilon_{A}+1} \int d^{2} \theta \theta^{2}\left(\sigma_{\alpha}\right)_{B}{ }^{A} \frac{\partial^{2} \delta_{L}}{\partial \theta^{2} \delta \Phi^{A}(\theta)} \frac{\delta}{\delta \bar{\Phi}_{B}(\theta)}=(-1)^{\epsilon_{A}}\left(\sigma_{\alpha}\right)_{B}{ }^{A} \frac{\delta_{L}}{\delta \phi^{A}} \frac{\delta}{\delta \eta_{B}}
$$

As long as $m \neq 0$ the operators $\bar{\Delta}_{m}^{a}$ are neither nilpotent nor do they anticommute among themselves; instead, together with the operators $\bar{\Delta}_{\alpha}$ they generate a superalgebra isomorphic to $\operatorname{osp}(1,2)$ :

$$
\begin{aligned}
{\left[V_{\alpha}, V_{\beta}\right] } & =\epsilon_{\alpha \beta}^{\gamma} V_{\gamma}, & {\left[\bar{\Delta}_{\alpha}, \bar{\Delta}_{\beta}\right] } & =(i / \hbar) \epsilon_{\alpha \beta}^{\gamma} \bar{\Delta}_{\gamma}, \\
{\left[V_{\alpha}, V_{m}^{a}\right] } & =V_{m}^{b}\left(\sigma_{\alpha}\right)_{b}^{a}, & {\left[\bar{\Delta}_{\alpha}, \bar{\Delta}_{m}^{a}\right] } & =(i / \hbar) \bar{\Delta}_{m}^{b}\left(\sigma_{\alpha}\right)_{b}{ }^{a}, \\
\left\{V_{m}^{a}, V_{m}^{b}\right\} & =-m^{2}\left(\sigma^{\alpha}\right)^{a b} V_{\alpha}, & \left\{\bar{\Delta}_{m}^{a}, \bar{\Delta}_{m}^{b}\right\} & =-(i / \hbar) m^{2}\left(\sigma^{\alpha}\right)^{a b} \bar{\Delta}_{\alpha} .
\end{aligned}
$$


The $m$-dependent quantum action $W_{m}\left(\Phi^{A}(\theta), \bar{\Phi}_{A}(\theta)\right)$ is required to obey the $m$-extended generalized quantum master equations

$$
\bar{\Delta}_{m}^{a} \exp \left\{(i / \hbar) W_{m}\right\}=0 \quad \Longleftrightarrow \quad \frac{1}{2}\left(W_{m}, W_{m}\right)^{a}+V_{m}^{a} W_{m}=i \hbar \Delta^{a} W_{m}
$$

which ensure (anti)BRST invariance, and the generating equations of $S p(2)$-invariance:

$$
\bar{\Delta}_{\alpha} \exp \left\{(i / \hbar) W_{m}\right\}=0 \quad \Longleftrightarrow \quad \frac{1}{2}\left\{W_{m}, W_{m}\right\}_{\alpha}+V_{\alpha} W_{m}=i \hbar \Delta_{\alpha} W_{m}
$$

where the curly superbrackets $\{F, G\}_{\alpha}$ are defined by

$$
\{F, G\}_{\alpha}=-\int d^{2} \theta\left\{\theta^{2} \frac{\partial^{2} \delta F}{\partial \theta^{2} \delta \Phi^{A}(\theta)} \frac{\delta G}{\delta \bar{\Phi}_{B}(\theta)}\left(\sigma_{\alpha}\right)_{B}{ }^{A}+(-1)^{\epsilon(F) \epsilon(G)}(F \leftrightarrow G)\right\} .
$$

The gauge fixed quantum action $W_{m, \text { ext }}\left(\Phi^{A}(\theta), \bar{\Phi}_{A}(\theta)\right)$ is introduced according to

$$
\exp \left\{(i / \hbar) W_{m, \text { ext }}\right\}=\hat{U}_{m}(F) \exp \left\{(i / \hbar) W_{m}\right\}
$$

where the operator $\hat{U}_{m}(F)$ has to be choosen as 8

$$
\hat{U}_{m}(F)=\exp \left\{(\hbar / i) \hat{T}_{m}(F)\right\} \quad \text { with } \quad \hat{T}_{m}(F)=\frac{1}{2} \epsilon_{a b}\left\{\bar{\Delta}_{m}^{b},\left[\bar{\Delta}_{m}^{a}, F\right]\right\}+(i / \hbar)^{2} m^{2} F
$$

$F=F\left(\Phi^{A}(\theta)\right)$ being the gauge fixing functional. With these definitions one establishes the following two relations:

$$
\begin{gathered}
{\left[\bar{\Delta}_{m}^{a}, \hat{T}_{m}(F)\right]=\frac{1}{2}(i / \hbar)\left(\sigma^{\alpha}\right)_{b}^{a}\left[\bar{\Delta}_{m}^{b},\left[\bar{\Delta}_{\alpha}, F\right]\right]} \\
{\left[\bar{\Delta}_{\alpha}, \hat{T}_{m}(F)\right]=\frac{1}{2} \epsilon_{a b}\left\{\bar{\Delta}_{m}^{b},\left[\bar{\Delta}_{m}^{a},\left[\bar{\Delta}_{\alpha}, F\right]\right]\right\}+(i / \hbar)^{2} m^{2}\left[\bar{\Delta}_{\alpha}, F\right] .}
\end{gathered}
$$

Restricting $F\left(\Phi^{A}(\theta)\right)$ to be a $S p(2)$-scalar by imposing the condition $\left[\bar{\Delta}_{\alpha}, F\right] W_{m}=0$ it can be verified (see Ref. [B]) that the commutators $\left[\bar{\Delta}_{m}^{a}, \hat{U}_{m}(F)\right]$ and $\left[\bar{\Delta}_{\alpha}, \hat{U}_{m}(F)\right]$, if applied on $\exp \left\{(i / \hbar) W_{m}\right\}$, vanish on the subspace of admissible actions $W_{m}$. These action are determined by the condition

$$
\int d^{2} \theta \theta^{2}\left\{\frac{\delta W_{m}}{\delta \bar{\Phi}_{A}(\theta)}+\Phi^{A}(\theta)\right\}=0 \quad \Longleftrightarrow \quad \frac{\delta W_{m}}{\delta \eta_{A}}=\phi^{A},
$$

i.e., depending only linearly on $\eta_{A}$. This condition ensures that the gauge fixed quantum action $W_{m, \text { ext }}$ also satisfies the quantum master equations (4.9) and (4.10). Then, by virtue of (4.13), the restriction $\left[\bar{\Delta}_{\alpha}, F\right] W_{m}=0$ becomes

$$
\left[\bar{\Delta}_{\alpha}, F\right] W_{m}=0 \quad \Longrightarrow \quad \int d^{2} \theta \theta^{2} \frac{\partial^{2} \delta F}{\partial \theta^{2} \delta \Phi^{A}(\theta)} \Phi^{B}(\theta)\left(\sigma_{\alpha}\right)_{B}{ }^{A}+V_{\alpha} F=0
$$


which expresses the $S p(2)$-invariance of $F$. Furthermore, the quantum master equations (4.10) simplify into

$$
\bar{\Delta}_{\alpha} \exp \left\{(i / \hbar) W_{m}\right\}=0 \quad \Longrightarrow \quad \int d^{2} \theta \theta^{2} \frac{\partial^{2} \delta W_{m}}{\partial \theta^{2} \delta \Phi^{A}(\theta)} \Phi^{B}(\theta)\left(\sigma_{\alpha}\right)_{B}{ }^{A}+V_{\alpha} W_{m}=0,
$$

since the $\sigma_{\alpha}-$ matrices are traceless.

The equations (4.14) for $\alpha=0$ express the ghost number conservation of the action $W_{m}, \operatorname{gh}\left(W_{m}\right)=0$. Thereby the ghost numbers of the fields and antifields are given by

$$
\begin{aligned}
& \operatorname{gh}\left(\phi^{A}\right)=-\left(0, \sum_{r=1}^{s}(-1)^{a_{r}}, \sum_{r=0}^{s}(-1)^{a_{r}}\right), \quad \text { where } \quad a_{r}=1,2, \\
& \operatorname{gh}\left(\bar{\phi}_{A}\right)=-\operatorname{gh}\left(\phi^{A}\right), \quad \operatorname{gh}\left(\phi_{A a}^{*}\right)=-\operatorname{gh}\left(\phi^{A}\right)+(-1)^{a}, \quad \operatorname{gh}\left(\eta_{A}\right)=-\operatorname{gh}\left(\phi^{A}\right) .
\end{aligned}
$$

(C) New ghost number conservation

In Ref. 13 also a so-called new ghost number was ascribed to all fields and antifields of the solutions of the classical master equations in the following way:

$$
\begin{aligned}
\operatorname{ngh}\left(\phi^{A}\right) & =(0, s+2, s+1), \\
\operatorname{ngh}\left(\bar{\phi}_{A}\right)=-\operatorname{ngh}\left(\phi^{A}\right)-2, \quad \operatorname{ngh}\left(\phi_{A a}^{*}\right) & =-\operatorname{ngh}\left(\phi^{A}\right)-1, \quad \operatorname{ngh}\left(\eta_{A}\right)=-\operatorname{ngh}\left(\phi^{A}\right) .
\end{aligned}
$$

According to these definitions we also have ngh $\left(\theta^{a}\right)=-1$. In comparison with Eqs. (2.12) and (2.13) it follows that the new ghost number agrees with the Weyl weight of the fields and antifields, i.e.,

$$
\begin{aligned}
& \operatorname{ngh}\left(\phi^{A}\right)=\alpha\left(\phi^{A}\right), \quad \operatorname{ngh}\left(\pi^{A a}\right)=\alpha\left(\pi^{A a}\right), \quad \operatorname{ngh}\left(\lambda^{A}\right)=\alpha\left(\lambda^{A}\right), \\
& \operatorname{ngh}\left(\bar{\phi}_{A}\right)=\alpha\left(\bar{\phi}_{A}\right), \quad \operatorname{ngh}\left(\phi_{A a}^{*}\right)=\alpha\left(\phi_{A a}^{*}\right), \quad \operatorname{ngh}\left(\eta_{A}\right)=\alpha\left(\eta_{A}\right) \text {. }
\end{aligned}
$$

In order to clarify how in our approach both numbers are related to each other let us introduce the following differential operator

$$
\bar{\Delta}_{m}=\Delta+(i / \hbar) V_{m}, \quad V_{m} \equiv V+m \frac{\partial}{\partial m},
$$

with the dilatations $V$ given by Eq. (3.22) and the (second-order) differential operator $\Delta$ defined by

$$
\Delta=(-1)^{\epsilon_{A}+1} \int d^{2} \theta \theta^{2} \gamma_{B}^{A} \frac{\partial^{2} \delta_{L}}{\partial \theta^{2} \delta \Phi^{A}(\theta)} \frac{\delta}{\delta \bar{\Phi}_{B}(\theta)}=(-1)^{\epsilon_{A}} \gamma_{B}^{A} \frac{\delta_{L}}{\delta \phi^{A}} \frac{\delta}{\delta \eta_{B}}
$$

The new operator $\bar{\Delta}_{m}$ together with the generating operators $\bar{\Delta}_{m}^{a}$ and $\bar{\Delta}_{\alpha}$ form an extension of the $\operatorname{osp}(1,2)$-superalgebra being isomorphic to $\operatorname{ssp}(1,2) \oplus u(1)$ where, in addition 
to the (anti)commutation relations (4.8), the following relations hold true:

$$
\begin{aligned}
{\left[V_{m}, V_{m}\right] } & =0, & & {\left[\bar{\Delta}_{m}, \bar{\Delta}_{m}\right]=0, } \\
{\left[V_{m}, V_{\alpha}\right] } & =0, & {\left[\bar{\Delta}_{m}, \bar{\Delta}_{\alpha}\right] } & =0, \\
{\left[V_{m}, V_{m}^{a}\right] } & =V_{m}^{a}, & & {\left[\bar{\Delta}_{m}, \bar{\Delta}_{m}^{a}\right]=(i / \hbar) \bar{\Delta}_{m}^{a} . }
\end{aligned}
$$

Let us assume now that solutions $W_{m}$ of the quantum master equations (4.10) and (4.11) can be constructed which also satisfy the following equation:

$$
\bar{\Delta}_{m} \exp \left\{(i / \hbar) W_{m}\right\}=0 \quad \Longleftrightarrow \quad \frac{1}{2}\left\{W_{m}, W_{m}\right\}+V_{m} W_{m}=i \hbar \Delta_{m} W_{m}
$$

with the following abbreviation

$$
\{F, G\}=-\int d^{2} \theta\left\{\theta^{2} \frac{\partial^{2} \delta F}{\partial \theta^{2} \delta \Phi^{A}(\theta)} \frac{\delta G}{\delta \bar{\Phi}_{B}(\theta)} \gamma_{B}^{A}+(-1)^{\epsilon(F) \epsilon(G)}(F \leftrightarrow G)\right\}
$$

Notice, that $\{F, G\}$ does not define a new superbracket since $\gamma_{B}^{A}=\delta_{B}^{A} \alpha\left(\phi^{A}\right)$ is a diagonal matrix. Taking into account the restriction (4.13) the additional master equation (4.17), at the lowest order of $\hbar$, simplifies according to

$$
\int d^{2} \theta \theta^{2} \frac{\partial^{2} \delta S_{m}}{\partial \theta^{2} \delta \Phi^{A}(\theta)} \Phi^{B}(\theta) \gamma_{B}^{A}+V_{m} S_{m}=0
$$

Obviously, the matrix $\gamma_{A}^{B}$ is uniquely determined by solving the quantum master equations (4.9) and (4.10) at the lowest order of $\hbar$, together with Eq. (4.19). The matrix $\bar{\gamma}_{A}^{B}$, which enters in $V_{m}$, is fixed by the requirement (2.10). Equation (4.19) expresses the conservation of the new ghost number of $S_{m}$ in the case $m \neq 0$, i.e., $\operatorname{ngh}\left(S_{m}\right)=0$. Thereby, we have formally ascribed also a new ghost number resp. Weyl weight to the mass parameter $m$, namely, according to the definition of $V_{m}, \operatorname{ngh}(m)=1$ resp. $\alpha(m)=1$. This already has been used in the definition of $V_{m}^{a}$, Eq. (4.6).

Let us emphasize that the equation (4.17) is quite formal since its right-hand side, for the same reasons as explained in the Introduction, is not well defined. Since, generally, the new ghost number is conserved only in the classical limit, we restricted ourselves in (4.19) to the lowest order approximation. In order to express the new ghost number conservation to higher orders - which is, of course, only possible as long as the dilatation invariance in superspace is not broken by radiative corrections - this requires a sensitive definition of the expression $\Delta_{m} W_{m}$ on the right-hand side of Eq. (4.17). In order to obtain a corresponding local quantum operator equation, which is valid to all orders in perturbation theory, one can use the method described in Ref. [10]. 
Independently, by introducing a gauge the gauge-fixed quantum action (4.12) breaks the new ghost number conservation. Namely, because of

$$
\left[\Delta_{m}, \hat{T}_{m}(F)\right]=\frac{1}{2} \epsilon_{a b}\left\{\bar{\Delta}_{m}^{b},\left[\bar{\Delta}_{m}^{a},\left[\Delta_{m}, F\right]+2 F\right]\right\}+(i / \hbar)^{2} m^{2}\left(\left[\Delta_{m}, F\right]+2 F\right),
$$

the action (4.12) is only a solution of (4.19) iff

$$
\left[\Delta_{m}, F\right] W_{m}=-2 F \quad \Longrightarrow \quad \int d^{2} \theta \theta^{2} \frac{\partial^{2} \delta F}{\partial \theta^{2} \delta \Phi^{A}(\theta)} \Phi^{B}(\theta) \gamma_{B}^{A}+V_{m} F=-2 F,
$$

where the second equation follows from the first one by taking into account the condition (4.13). On the other hand, the expression on the left-hand side (modulo the signum of $F$ ), can never be negative, since $F$ depends only on $\Phi^{A}$ which has positive Weyl weight,

$$
\operatorname{sgn}(F)\left\{\int d^{2} \theta \theta^{2} \frac{\partial^{2} \delta F}{\partial \theta^{2} \delta \Phi^{A}(\theta)} \Phi^{B}(\theta) \gamma_{B}^{A}+V_{m} F\right\} \geq 0 .
$$

This proves that the new ghost number conservation is broken through gauge fixing.

\section{Generating functionals and gauge (in)dependence}

Next, we turn to the question of gauge (in)dependence of the generating functionals of Green's functions [13, 8].

(A) $S p(2)$-covariant approach

In discussing this question it is convenient to study first the symmetry properties of the vacuum functional $Z(0)$ defined as

$$
Z(0)=\int d \Phi^{A}(\theta) d \bar{\Phi}_{A}(\theta) \rho\left(\bar{\Phi}_{A}(\theta)\right) \exp \left\{(i / \hbar)\left(W_{\mathrm{ext}}+S_{X}\right)\right\} .
$$

Here, $\rho\left(\bar{\Phi}_{A}(\theta)\right)$ is a density having the form of a $\delta$-functional,

$$
\rho\left(\bar{\Phi}_{A}(\theta)\right)=\delta\left(\int d^{2} \theta \bar{\Phi}_{A}(\theta)\right)
$$

and $S_{X}$ is given by

$$
S_{X}=\int d^{2} \theta \bar{\Phi}_{A}(\theta) \Phi^{A}(\theta)
$$

The term $S_{X}$ can be cast into the (anti)BRST-invariant form

$$
S_{X}=\frac{1}{2} \epsilon_{a b}\left(V^{b}\left(V^{a} X-X U^{a}\right)+\left(V^{a} X-X U^{a}\right) U^{b}\right), \quad X \equiv-\int d^{2} \theta \theta^{2} \bar{\Phi}_{A}(\theta) \Phi^{A}(\theta),
$$

with $V^{a} \equiv V_{+}^{a}$ and $U^{a} \equiv U_{+}^{a}$, whose action on $\bar{\Phi}_{A}(\theta)$ and $\Phi^{A}(\theta)$ are defined in Eqs. (3.19) and (3.24), respectively, satisfying $\left\{V^{a}, V^{b}\right\}=0$ and $\left\{U^{a}, U^{b}\right\}=0$. Let us combine the action of $V^{a}$ and $U^{a}$ on an arbitrary functional $Y$ according to

$$
L^{a} Y \equiv V^{a} Y-(-1)^{\epsilon(Y)} Y U^{a}, \quad\left\{L^{a}, L^{b}\right\}=0,
$$


then the operators $L^{a}$ are nilpotent and anticommuting.

Inserting into expression (5.1) the relation (4.5) and integrating by parts this gives

$$
Z(0)=\int d \Phi^{A}(\theta) d \bar{\Phi}_{A}(\theta) \rho\left(\bar{\Phi}_{A}(\theta)\right) \exp \left\{(i / \hbar)\left(W+S_{X}+S_{F}\right)\right\}
$$

with the following expression for $S_{F}$ :

$$
S_{F}=-\int d^{2} \theta\left\{\frac{\delta F}{\delta \Phi^{A}(\theta)} \frac{\partial^{2} \Phi^{A}(\theta)}{\partial \theta^{2}}+\frac{1}{2} \epsilon_{a b} \int d^{2} \bar{\theta} \frac{\partial \Phi^{A}(\theta)}{\partial \theta_{a}} \frac{\delta^{2} F}{\delta \Phi^{A}(\theta) \delta \Phi^{B}(\bar{\theta})} \frac{\partial \Phi^{B}(\bar{\theta})}{\partial \bar{\theta}_{b}}\right\} .
$$

This may be cast also into the (anti)BRST invariant form

$$
S_{F}=\frac{1}{2} \epsilon_{a b} F U^{b} U^{a}
$$

Then, by virtue of $L^{a} S_{X}=0$ and $L^{a} S_{F}=0$, it can be checked that the integrand of the vacuum functional (5.4) is invariant under the following global (anti)BRST transformations (thereby, one has to make use of Eq. (4.7)):

$$
\delta \Phi^{A}(\theta)=\Phi^{A}(\theta) U^{a} \mu_{a}, \quad \delta \bar{\Phi}_{A}(\theta)=\mu_{a} V^{a} \bar{\Phi}_{A}(\theta)+\mu_{a}\left(W, \bar{\Phi}_{A}(\theta)\right)^{a},
$$

where $\mu_{a}, \epsilon\left(\mu_{a}\right)=1$, is a $S p(2)$-doublet of constant anticommuting parameters. Here, we have taken into account that the density $\rho\left(\bar{\Phi}_{A}(\theta)\right)=\delta\left(\eta_{A}\right)$ is invariant under the transformations (5.6). These transformations realize the (anti)BRST symmetry in the superfield approach to quantum gauge theory.

The invariance of $Z(0)$ under the transformations (5.6) permits to study the question whether $Z(0)$ is independent on the choice of the gauge. Indeed, let us change the gaugefixing functional $F \rightarrow F+\delta F$. Then, the gauge-fixing term $S_{F}$ changes according to

$$
S_{F} \rightarrow S_{F+\delta F}=S_{F}+S_{\delta F}, \quad S_{\delta F}=\frac{1}{2} \epsilon_{a b}(\delta F) U^{b} U^{a}
$$

Now, we perform in the vacuum functional (5.4) the transformations (5.6) and choose the parameters $\mu_{a}$ as follows,

$$
\mu_{a}=-(i / \hbar) \frac{1}{2} \epsilon_{a b}(\delta F) U^{b}
$$

Thereby we induce the factor $\exp \left(\mu_{a} U^{a}\right)$ in the integration measure. Combining its exponent with $S_{F}$ leads to

$$
S_{F} \rightarrow S_{F}+(\hbar / i) \mu_{a} U^{a}=S_{F}-\frac{1}{2} \epsilon_{a b}(\delta F) U^{b} U^{a}=S_{F}-S_{\delta F}
$$

By comparison with (5.7) this proves that the vacuum functional and, therefore, also the $S$-matrix is independent on the choice of the gauge. 
(B) osp(1,2)-covariant approach

In this approach the vacuum functional $Z_{m}(0)$, which depends on the additional mass parameter $m$, is defined as

$$
Z_{m}(0)=\int d \Phi^{A}(\theta) d \bar{\Phi}_{A}(\theta) \rho\left(\bar{\Phi}_{A}(\theta)\right) \exp \left\{(i / \hbar)\left(W_{m, \mathrm{ext}}+S_{m, X}\right)\right\},
$$

with

$$
S_{m, X}=S_{X}+m^{2} \int d^{2} \theta \theta^{2} \bar{\Phi}_{A}(\theta) \gamma_{B}^{A} \Phi^{B}(\theta),
$$

where $S_{X}$ again is given by Eq. (5.3). The term $S_{m, X}$ can be rewritten as

$$
S_{m, X}=\frac{1}{2} \epsilon_{a b}\left(V_{m}^{b}\left(V_{m}^{a} X-X U_{m}^{a}\right)+\left(V_{m}^{a} X-X U_{m}^{a}\right) U_{m}^{b}\right)+m^{2} X, \quad V_{\alpha} X+X U_{\alpha}=0,
$$

with $\left(V_{m}^{a} \equiv V_{+}^{a}+\frac{1}{2} m^{2} V_{-}^{a}, V_{\alpha}\right)$ and $\left(U_{m}^{a} \equiv U_{+}^{a}+\frac{1}{2} m^{2} U_{-}^{a}, U_{\alpha}\right)$ obeying the following $\operatorname{osp}(1,2)-$ superalgebras

$$
\begin{aligned}
{\left[V_{\alpha}, V_{\beta}\right] } & =\epsilon_{\alpha \beta}^{\gamma} V_{\gamma}, & {\left[U_{\alpha}, U_{\beta}\right] } & =-\epsilon_{\alpha \beta}^{\gamma} U_{\gamma}, \\
{\left[V_{\alpha}, V_{m}^{a}\right] } & =V_{m}^{b}\left(\sigma_{\alpha}\right)_{b}^{a}, & {\left[U_{\alpha}, U_{m}^{a}\right] } & =-U_{m}^{b}\left(\sigma_{\alpha}\right)_{b}{ }^{a}, \\
\left\{V_{m}^{a}, V_{m}^{b}\right\} & =-m^{2}\left(\sigma^{\alpha}\right)^{a b} V_{\alpha}, & \left\{U_{m}^{a}, U_{m}^{b}\right\} & =m^{2}\left(\sigma^{\alpha}\right)^{a b} U_{\alpha},
\end{aligned}
$$

respectively; the action of $\left(V_{ \pm}^{a}, V_{\alpha}\right)$ and $\left(U_{ \pm}^{a}, U_{\alpha}\right)$ on $\bar{\Phi}_{A}(\theta)$ and $\Phi^{A}(\theta)$ are defined by Eqs. (3.20)-(3.22) and (3.25)-(3.27), respectively.

Inserting into the expression (5.8) the relation (4.12) and integrating by parts this yields

$$
Z_{m}(0)=\int d \Phi^{A}(\theta) d \bar{\Phi}_{A}(\theta) \rho\left(\bar{\Phi}_{A}(\theta)\right) \exp \left\{(i / \hbar)\left(W_{m}+S_{m, X}+S_{m, F}\right)\right\},
$$

with

$$
S_{m, F}=S_{F}-\frac{1}{2} m^{2} \int d^{2} \theta \theta^{2} \frac{\partial^{2} \delta F}{\partial \theta^{2} \delta \Phi^{A}(\theta)} \gamma_{B}^{A} \Phi^{B}(\theta),
$$

where $S_{F}$ is given by Eq. (5.5). The gauge-fixing term $S_{m, F}$ can be rewritten as

$$
S_{m, F}=\frac{1}{2} \epsilon_{a b} F U_{m}^{b} U_{m}^{a}+m^{2} F
$$

Let us now introduce the differential operators

$$
L_{m}^{a} Y \equiv V_{m}^{a} Y-(-1)^{\epsilon(Y)} Y U_{m}^{a}, \quad L_{\alpha} Y \equiv V_{\alpha} Y+Y U_{\alpha}
$$


which, by virtue of the relations (5.10), satisfy the $\operatorname{osp}(1,2)$-superalgebra

$$
\left[L_{\alpha}, L_{\beta}\right]=\epsilon_{\alpha \beta}^{\gamma} L_{\gamma}, \quad\left[L_{\alpha}, L_{m}^{a}\right]=L_{m}^{b}\left(\sigma_{\alpha}\right)_{b}^{a}, \quad\left\{L_{m}^{a}, L_{m}^{b}\right\}=-m^{2}\left(\sigma^{\alpha}\right)^{a b} L_{\alpha}
$$

By using this algebra, after tedious but straightforward computations, one verifies the following relations:

$$
L_{m}^{c}\left(\frac{1}{2} \epsilon_{a b} L_{m}^{b} L_{m}^{a}+m^{2}\right)=\frac{1}{2} m^{2}\left(\sigma^{\alpha}\right)_{d}^{c} L_{m}^{d} L_{\alpha}, \quad\left[L_{\alpha}, \frac{1}{2} \epsilon_{a b} L_{m}^{b} L_{m}^{a}+m^{2}\right]=0 .
$$

Therefore, it holds $L_{m}^{a} S_{m, X}=0$ and $L_{\alpha} S_{m, F}=0$, since $X$ and $F$ are $S p(2)$-invariant. Because $W_{m}$ exhibits the same $\eta$-dependence as $-S_{m, X}$, Eqs. (4.13), (5.3) and (5.9), $W_{m}+S_{m, X}$ is independent on $\eta_{A}$ and, hence, the integration over $\bar{\Phi}_{A}$ with the density $\rho\left(\bar{\Phi}_{A}(\theta)\right)=\delta\left(\eta_{A}\right)$ yields a constant factor which is equal to one.

We assert now that the integrand in (5.11) is invariant under the following global transformations (thereby, one has to make use of the Eqs. (4.9) and (4.10), respectively):

$$
\begin{array}{ll}
\delta \Phi^{A}(\theta)=\Phi^{A}(\theta) U_{m}^{a} \mu_{a}, & \delta \bar{\Phi}_{A}(\theta)=\mu_{a} V_{m}^{a} \bar{\Phi}_{A}(\theta)+\mu_{a}\left(W_{m}, \bar{\Phi}_{A}(\theta)\right)^{a} \\
\delta \Phi^{A}(\theta)=\Phi^{A}(\theta) U_{\alpha} \mu^{\alpha}, & \delta \bar{\Phi}_{A}(\theta)=\mu^{\alpha} V_{\alpha} \bar{\Phi}_{A}(\theta)+\mu^{\alpha}\left\{W_{m}, \bar{\Phi}_{A}(\theta)\right\}_{\alpha},
\end{array}
$$

where $\mu_{a}, \epsilon\left(\mu_{a}\right)=1$, and $\mu^{\alpha}, \epsilon\left(\mu^{\alpha}\right)=0$, are constant anticommuting resp. commuting parameters. Notice, that in the present case $\rho\left(\bar{\Phi}_{A}(\theta)\right)$ is not invariant under the transformations (5.12). The transformations (5.12) and (5.13) realize the $m$-extended (anti)BRST- and $S p(2)$-symmetry, respectively.

Next, we study the question whether the mass dependent terms in $Z_{m}(0)$ violate the independence on the choice of the gauge. Proceeding as in the previous case, by changing the gauge-fixing functional $F \rightarrow F+\delta F$ the gauge-fixing term changes according to

$$
S_{m, F} \rightarrow S_{m, F+\delta F}=S_{m, F}+S_{m, \delta F}, \quad S_{m, \delta F}=\frac{1}{2} \epsilon_{a b}(\delta F) U_{m}^{b} U_{m}^{a}+m^{2} \delta F .
$$

Now, carring out in (5.11) the transformations (5.12), we choose

$$
\mu_{a}=-(i / \hbar) \frac{1}{2} \epsilon_{a b}(\delta F) U_{m}^{b}
$$

which leads to

$$
S_{m, F} \rightarrow S_{m, F}+(\hbar / i) \mu_{a} U_{m}^{a}=S_{m, F}-\frac{1}{2} \epsilon_{a b}(\delta F) U_{m}^{b} U_{m}^{a}=S_{m, F}-S_{m, \delta F}+m^{2} \delta F
$$

By comparison with (5.14) we observe that the mass term $m^{2} F$ violates the independence of $Z_{m}(0)$ on the choice of the gauge. One may try to compensate this undesired term $m^{2} \delta F$ by means of an additional change of variables using the transformations (5.13). But this change should not destroy the form of the action arrived at the previous stage. However, such additional changes of variables lead to a Berezinian which is equal to one because $\sigma_{\alpha}$ are traceless. Thus, the unwanted term could never be compensated. 


\section{Irreducible and first-stage reducible massive theo- ries with closed algebra}

In the preceeding Sections we gave a general framework of quantizing massive general gauge theories by introducing on the space of superfields and superantifields a set of differential operators which obey the superalgebra $\operatorname{sl}(1,2)$. Thereby, we extended our previous work [8] on $\operatorname{osp}(1,2)$-covariant quantization where we already considered the case of irreducible and first-stage reducible gauge theories with closed algebra. In order to illustrate our present approach let us study how the construction of these theories is extended now. (Thereby we also simplify some of our former calculations.)

(A) Generic form of the dependence on the antifields

Our aim here is to construct a proper solution $S_{m}$ of the classical master equations

$$
\frac{1}{2}\left(S_{m}, S_{m}\right)^{a}+V_{m}^{a} S_{m}=0, \quad \frac{1}{2}\left\{S_{m}, S_{m}\right\}_{\alpha}+V_{\alpha} S_{m}=0, \quad \frac{1}{2}\left\{S_{m}, S_{m}\right\}+V_{m} S_{m}=0
$$

which are obtained from the quantum master equations (4.9), (4.10) and (4.17) at the lowest order approximation of $\hbar$. Let us rewrite more explicit the brackets in Eqs. (6.1) using their definitions, Eqs. (4.4), (4.11) and (4.18),

$\frac{\delta S_{m}}{\delta \phi^{A}} \frac{\delta S_{m}}{\delta \phi_{A a}^{*}}+V_{m}^{a} S_{m}=0, \quad \frac{\delta S_{m}}{\delta \phi^{A}} \frac{\delta S_{m}}{\delta \eta_{B}}\left(\sigma_{\alpha}\right)_{B}{ }^{A}+V_{\alpha} S_{m}=0, \quad \frac{\delta S_{m}}{\delta \phi^{A}} \frac{\delta S_{m}}{\delta \eta_{B}} \gamma_{A}^{B}+V_{m} S_{m}=0$

with $V_{m}^{a} \equiv V_{+}^{a}+\frac{1}{2} m^{2} V_{-}^{a}$ and $V_{m} \equiv V+m \partial / \partial m$, where the action of $V_{ \pm}^{a}, V_{\alpha}$ and $V$ on the antifields is given by (see Eqs. (2.7) and (2.8)):

$$
\begin{aligned}
V_{+}^{a} & =\epsilon^{a b} \phi_{A b}^{*} \frac{\delta}{\delta \bar{\phi}_{A}}-\eta_{A} \frac{\delta}{\delta \phi_{A a}^{*}}, \\
V_{-}^{a} & =\bar{\phi}_{B}\left(\left(\sigma^{\alpha}\right)_{b}^{a}\left(\sigma_{\alpha}\right)^{B}{ }_{A}-\delta_{b}^{a} \bar{\gamma}_{A}^{B}\right) \frac{\delta}{\delta \phi_{A b}^{*}}+\phi_{B b}^{*}\left(\left(\sigma^{\alpha}\right)^{a b}\left(\sigma_{\alpha}\right)^{B}{ }_{A}-\epsilon^{a b}\left(\bar{\gamma}_{A}^{B}+2 \delta_{A}^{B}\right)\right) \frac{\delta}{\delta \eta_{A}}, \\
V_{\alpha} & =\bar{\phi}_{B}\left(\sigma_{\alpha}\right)^{B}{ }_{A} \frac{\delta}{\delta \bar{\phi}_{A}}+\left(\phi_{B b}^{*}\left(\sigma_{\alpha}\right)^{B}{ }_{A}+\phi_{A a}^{*}\left(\sigma_{\alpha}\right)^{a}{ }_{b}\right) \frac{\delta}{\delta \phi_{A b}^{*}}+\eta_{B}\left(\sigma_{\alpha}\right)^{B}{ }_{A} \frac{\delta}{\delta \eta_{A}}, \\
V & =\bar{\phi}_{B} \bar{\gamma}_{A}^{B} \frac{\delta}{\delta \bar{\phi}_{A}}+\phi_{B b}^{*}\left(\bar{\gamma}_{A}^{B}+\delta_{A}^{B}\right) \frac{\delta}{\delta \phi_{A b}^{*}}+\eta_{B}\left(\bar{\gamma}_{A}^{B}+2 \delta_{A}^{B}\right) \frac{\delta}{\delta \eta_{A}} .
\end{aligned}
$$

The symmetry properties (6.2) of $S_{m}$ may be expressed also by the following equations:

$$
\mathbf{s}_{m}^{a} S_{m}=0 \quad \mathbf{d}_{\alpha} S_{m}=0, \quad \mathbf{d}_{m} S_{m}=0
$$

with $\mathbf{s}_{m}^{a} \equiv \mathbf{s}_{+}^{a}+\frac{1}{2} m^{2} \mathbf{s}_{-}^{a}$ and $\mathbf{d}_{m} \equiv \mathbf{d}+m \partial / \partial m$, where the operators $\mathbf{s}_{ \pm}^{a}, \mathbf{d}_{\alpha}$ and $\mathbf{d}$ are 
required to fulfil the $s l(1,2)$-superalgebra:

$$
\begin{aligned}
& {\left[\mathbf{d}, \mathbf{d}_{\alpha}\right]=0, \quad\left[\mathbf{d}, \mathbf{s}_{+}^{a}\right]=\mathbf{s}_{+}^{a}, \quad\left[\mathbf{d}, \mathbf{s}_{-}^{a}\right]=-\mathbf{s}_{-}^{a},} \\
& {\left[\mathbf{d}_{\alpha}, \mathbf{d}_{\beta}\right]=\epsilon_{\alpha \beta}^{\gamma} \mathbf{d}_{\gamma}, \quad\left[\mathbf{d}_{\alpha}, \mathbf{s}_{+}^{a}\right]=\mathbf{s}_{+}^{b}\left(\sigma_{\alpha}\right)_{b}^{a}, \quad\left[\mathbf{d}_{\alpha}, \mathbf{s}_{-}^{a}\right]=\mathbf{s}_{-}^{b}\left(\sigma_{\alpha}\right)_{b}^{a},} \\
& \left\{\mathbf{s}_{+}^{a}, \mathbf{s}_{+}^{b}\right\}=0, \quad\left\{\mathbf{s}_{-}^{a}, \mathbf{s}_{-}^{b}\right\}=0, \quad\left\{\mathbf{s}_{+}^{a}, \mathbf{s}_{-}^{b}\right\}=-\left(\sigma^{\alpha}\right)^{a b} \mathbf{d}_{\alpha}-\epsilon^{a b} \mathbf{d} .
\end{aligned}
$$

Indeed, let us restrict our considerations to solutions $S_{m}$ being linear with respect to the antifields. Let us remark that proper solutions of the classical master equations for theories with closed gauge algebra and vanishing new ghost number depends only linearly on the antifields [16]. Such solutions can be written in the form [8]

$$
S_{m}=S_{\mathrm{cl}}+\left(\frac{1}{2} \epsilon_{a b} \mathbf{s}_{m}^{b} \mathbf{s}_{m}^{a}+m^{2}\right) X,
$$

where $X$ is assumed to be a $S p(2)$-scalar (in fact the only one we are able to build up linear in the antifields) and, in accordance with the requirement (2.10), to have Weyl weight $\alpha(X)=\alpha\left(\bar{\phi}_{A}\right)+\alpha\left(\phi^{A}\right)=-2$,

$$
X=\bar{\phi}_{A} \phi^{A} \quad \text { with } \quad \mathbf{d}_{\alpha} X=0, \quad \mathbf{d}_{m} X=-2 X .
$$

Then, by making use of the $\operatorname{ssp}(1,2) \oplus u(1)$-superalgebra of these symmetry operators,

$$
\begin{aligned}
& {\left[\mathbf{d}_{m}, \mathbf{d}_{m}\right]=0, \quad\left[\mathbf{d}_{m}, \mathbf{d}_{\alpha}\right]=0, \quad\left[\mathbf{d}_{m}, \mathbf{s}_{m}^{a}\right]=\mathbf{s}_{m}^{a},} \\
& {\left[\mathbf{d}_{\alpha}, \mathbf{d}_{\beta}\right]=\epsilon_{\alpha \beta}{ }^{\gamma} \mathbf{d}_{\gamma}, \quad\left[\mathbf{d}_{\alpha}, \mathbf{s}_{m}^{a}\right]=\mathbf{s}_{m}^{b}\left(\sigma_{\alpha}\right)_{b}^{a}, \quad\left\{\mathbf{s}_{m}^{a}, \mathbf{s}_{m}^{b}\right\}=-m^{2}\left(\sigma^{\alpha}\right)^{a b} \mathbf{d}_{\alpha},}
\end{aligned}
$$

one establishes the following relations:

$$
\begin{aligned}
\mathbf{s}_{m}^{c}\left(\frac{1}{2} \epsilon_{a b} \mathbf{s}_{m}^{b} \mathbf{s}_{m}^{a}+m^{2}\right) & =\frac{1}{2} m^{2}\left(\sigma^{\alpha}\right)^{c}{ }_{d} \mathbf{s}_{m}^{d} \mathbf{d}_{\alpha}, \\
\mathbf{d}_{\alpha}\left(\frac{1}{2} \epsilon_{a b} \mathbf{s}_{m}^{b} \mathbf{s}_{m}^{a}+m^{2}\right) & =\left(\frac{1}{2} \epsilon_{a b} \mathbf{s}_{m}^{b} \mathbf{s}_{m}^{a}+m^{2}\right) \mathbf{d}_{\alpha}, \\
\mathbf{d}_{m}\left(\frac{1}{2} \epsilon_{a b} \mathbf{s}_{m}^{b} \mathbf{s}_{m}^{a}+m^{2}\right) & =\left(\frac{1}{2} \epsilon_{a b} \mathbf{s}_{m}^{b} \mathbf{s}_{m}^{a}+m^{2}\right)\left(\mathbf{d}_{m}+2\right) .
\end{aligned}
$$

From these relations, by virtue of (6.6), it follows that the ansatz (6.5) for $S_{m}$ really obeys the symmetry requirements (6.3). Thereby, it has to be taken into account that for the classical action $S_{\mathrm{cl}}(A)$ it holds $\mathbf{s}_{m}^{a} S_{\mathrm{cl}}(A)=0$ as well as $\mathbf{d}_{\alpha} S_{\mathrm{cl}}(A)=0$ and $\mathbf{d}_{m} S_{\mathrm{cl}}(A)=0$.

In order to convince ourselves that the equations (6.3) can be cast into the form (6.2) let us decompose $\mathbf{s}_{m}^{a}, \mathbf{d}_{\alpha}$ and $\mathbf{d}_{m}$ into a component acting on the fields and another one acting on the antifields as follows:

$$
\mathbf{s}_{m}^{a}=\left(\mathbf{s}_{m}^{a} \phi^{A}\right) \frac{\delta_{L}}{\delta \phi^{A}}+V_{m}^{a}, \quad \mathbf{d}_{\alpha}=\left(\mathbf{d}_{\alpha} \phi^{A}\right) \frac{\delta_{L}}{\delta \phi^{A}}+V_{\alpha}, \quad \mathbf{d}_{m}=\left(\mathbf{d}_{m} \phi^{A}\right) \frac{\delta_{L}}{\delta \phi^{A}}+V_{m} .
$$


The assumptions (6.6) are satisfied if the action of $\mathbf{d}_{\alpha}$ and $\mathbf{d}_{m}$ on $\phi^{A}$ is defined as

$$
\mathbf{d}_{\alpha} \phi^{A}=\phi^{B}\left(\sigma_{\alpha}\right)_{B}{ }^{A} \quad \text { and } \quad \mathbf{d}_{m} \phi^{A}=\phi^{B} \gamma_{B}^{A}
$$

Then from (6.5) one gets for $S_{m}$ the expression

$$
S_{m}=S_{\mathrm{cl}}+\left(\eta_{A}+\frac{1}{2} m^{2} \bar{\gamma}_{A}^{B} \bar{\phi}_{B}\right) \phi^{A}-\left(\mathbf{s}_{m}^{a} \phi^{A}\right) \phi_{A a}^{*}+\bar{\phi}_{A}\left(\frac{1}{2} \epsilon_{a b} \mathbf{s}_{m}^{b} \mathbf{s}_{m}^{a}+m^{2}\right) \phi^{A}
$$

with $\bar{\gamma}_{A}^{B}=-\gamma_{A}^{B}-2 \delta_{A}^{B}$. Now, performing in (6.3) the replacements $\mathbf{s}_{m}^{a} \phi^{A}=-\delta_{R} S_{m} / \delta \phi_{A a}^{*}$, $\mathbf{d}_{\alpha} \phi^{A}=\delta S_{m} / \delta \eta_{B}\left(\sigma_{\alpha}\right)_{B}{ }^{A}$ and $\mathbf{d}_{m} \phi^{A}=\delta S_{m} / \delta \eta_{B} \gamma_{B}^{A}$ it is easily seen that both symmetry requirements, Eqs. (6.2) and (6.3), are equivalent to each other. Thus, we are left with the exercise to determine the action of the $s l(1,2)$-superalgebra (6.4) on the components of the fields $\phi^{A}$. Thereby, we restrict ourselves to the cases of irreducible and first-stage reducible theories with closed gauge algebra.

(B) Explicit realization of sl(1,2) on the fields: Irreducible gauge theories

For irreducible theories with a closed algebra, because of $M_{\alpha_{0} \beta_{0}}^{i j}=0$, the algebra of the generators, Eq. (2.2), reduces to

$$
R_{\alpha_{0}, j}^{i} R_{\beta_{0}}^{j}-R_{\beta_{0}, j}^{i} R_{\alpha_{0}}^{j}=-R_{\gamma_{0}}^{i} F_{\alpha_{0} \beta_{0}}^{\gamma_{0}}
$$

where for the sake of simplicity we assume throughout this and the succeeding subsection that the $A^{i}$ are bosonic fields. This algebra defines the structure tensors $F_{\alpha_{0} \beta_{0}}^{\gamma_{0}}$. In general, the restrictions imposed by the Jacobi identity lead to additional equations with new structure tensors. But in the simple case under consideration it leads only to the following relation among the tensors $F_{\alpha_{0} \beta_{0}}^{\gamma_{0}}$ and the generators $R_{\alpha_{0}}^{i}$ :

$$
F_{\eta_{0} \alpha_{0}}^{\delta_{0}} F_{\beta_{0} \gamma_{0}}^{\eta_{0}}-R_{\alpha_{0}}^{i} F_{\beta_{0} \gamma_{0}, i}^{\delta_{0}}+\operatorname{cyclic} \operatorname{perm}\left(\alpha_{0}, \beta_{0}, \gamma_{0}\right)=0
$$

In order to construct the proper solution $S_{m}=S_{\mathrm{cl}}+\left(\frac{1}{2} \epsilon_{a b} \mathbf{s}_{m}^{b} \mathbf{s}_{m}^{a}+m^{2}\right) X$, Eq. (6.5), for $X$ one has to choose $X=\bar{A}_{i} A^{i}+\bar{B}_{\alpha_{0}} B^{\alpha_{0}}+\bar{C}_{\alpha a} C^{\alpha_{0} a}$. The $s l(1,2)$-transformations of the antifields $\bar{A}_{i}, \bar{B}_{\alpha_{0}}$ and $\bar{C}_{\alpha_{0} a}$ already has been given (see Appendix A). The corresponding nonlinear realization of the $s l(1,2)$ in terms of the fields $A^{i}, B^{\alpha_{0}}$ and $C^{\alpha_{0} a}$ reads (1) translations:

$$
\begin{aligned}
\mathbf{s}_{+}^{a} A^{i} & =R_{\alpha_{0}}^{i} C^{\alpha_{0} a}, \\
\mathbf{s}_{+}^{a} C^{\alpha_{0} b} & =\epsilon^{a b} B^{\alpha_{0}}-F_{\beta_{0} \gamma_{0}}^{\alpha_{0}} C^{\beta_{0} a} C^{\gamma_{0} b}, \\
\mathbf{s}_{+}^{a} B^{\alpha_{0}} & =\frac{1}{2} F_{\beta_{0} \gamma_{0}}^{\alpha_{0}} B^{\beta_{0}} C^{\gamma_{0} a}+\frac{1}{12} \epsilon_{c d}\left(F_{\eta_{0} \beta_{0}}^{\alpha_{0}} F_{\gamma_{0} \delta_{0}}^{\eta_{0}}+2 R_{\beta_{0}}^{i} F_{\gamma_{0} \delta_{0}, i}^{\alpha_{0}}\right) C^{\gamma_{0} a} C^{\delta_{0} c} C^{\beta_{0} d},
\end{aligned}
$$


(2) special conformal transformations:

$$
\begin{aligned}
\mathbf{s}_{-}^{a} A^{i} & =0, \\
\mathbf{s}_{-}^{a} C^{\alpha_{0} b} & =0, \\
\mathbf{s}_{-}^{a} B^{\alpha_{0}} & =-2 C^{\alpha_{0} a},
\end{aligned}
$$

(3) symplectic rotations:

$$
\begin{aligned}
\mathbf{d}_{\alpha} A^{i} & =0 \\
\mathbf{d}_{\alpha} C^{\alpha_{0} b} & =C^{\alpha_{0} a}\left(\sigma_{\alpha}\right)_{a}^{b}, \\
\mathbf{d}_{\alpha} B^{\alpha_{0}} & =0
\end{aligned}
$$

and (4) dilatations:

$$
\begin{aligned}
\mathbf{d} A^{i} & =0, \\
\mathbf{d} C^{\alpha_{0} b} & =C^{\alpha_{0} b}, \\
\mathbf{d} B^{\alpha_{0}} & =2 B^{\alpha_{0}} .
\end{aligned}
$$

By making use of Eqs. (6.8) and (6.9) it is a simple exercise to prove that the transformations (6.10)- 6.13) actually obey the $s l(1,2)$-superalgebra (6.4). Let us remark that the nonlinearity of the translations, Eqs. (6.10), is due to the fact that the components $\pi^{A a}$ and $\lambda^{a}$ of the superfield $\Phi^{A}(\theta)$ have been eliminated from the theory by integrating them out in Eq. (5.11).

(C) Explicit realization of sl(1,2) on the fields: First-stage reducible gauge theories

Now let us consider first-stage reducible theories. In that case, due to the condition of first-stage reducibility,

$$
R_{\alpha_{0}}^{i} Z_{\alpha_{1}}^{\alpha_{0}}=0
$$

there are independent zero-modes $Z_{\alpha_{1}}^{\alpha_{0}}$ of the generators $R_{\alpha_{0}}^{i}$. Their presence does not modify the gauge algebra

$$
R_{\alpha_{0}, j}^{i} R_{\beta_{0}}^{j}-R_{\beta_{0}, j}^{i} R_{\alpha_{0}}^{j}=-R_{\gamma_{0}}^{i} F_{\alpha_{0} \beta_{0}}^{\gamma_{0}}
$$

but it influences the solutions of the Jacobi identity which appears from the relation

$$
R_{\delta_{0}}^{j}\left(F_{\eta_{0} \alpha_{0}}^{\delta_{0}} F_{\beta_{0} \gamma_{0}}^{\eta_{0}}-R_{\alpha_{0}}^{i} F_{\beta_{0} \gamma_{0}, i}^{\delta_{0}}+\operatorname{cyclic} \operatorname{perm}\left(\alpha_{0}, \beta_{0}, \gamma_{0}\right)\right)=0
$$


In addition, new equations and structure tensors occure. One of these gauge structure relations is the reducibility condition (6.14) itself. In order to derive the others we proceed as follows:

First, let us cast the Jacobi identity (6.16) into a more practical form. Owing to (6.14) the expression in parenthesis must be proportional to the zero-modes $Z_{\alpha_{1}}^{\delta_{0}}$,

$$
F_{\eta_{0} \alpha_{0}}^{\delta_{0}} F_{\beta_{0} \gamma_{0}}^{\eta_{0}}-R_{\alpha_{0}}^{i} F_{\beta_{0} \gamma_{0}, i}^{\delta_{0}}+\text { cyclic perm }\left(\alpha_{0}, \beta_{0}, \gamma_{0}\right)=3 Z_{\alpha_{1}}^{\delta_{0}} H_{\alpha_{0} \beta_{0} \gamma_{0}}^{\alpha_{1}}
$$

where $H_{\alpha_{0} \beta_{0} \gamma_{0}}^{\alpha_{1}}(A)$ are new structure tensors being totally antisymmetric with respect to the indices $\alpha_{0}, \beta_{0}, \gamma_{0}$ and depending, in general, on the gauge fields $A^{i}$.

Next, we derive an expression for the combination $Z_{\alpha_{1}, j}^{\alpha_{0}} R_{\beta_{0}}^{j}$. Multiplying (6.15) by $Z_{\alpha_{1}}^{\alpha_{0}}$ and using the relation $R_{\alpha_{0}, j}^{i} Z_{\alpha_{1}}^{\alpha_{0}}=-R_{\alpha_{0}}^{i} Z_{\alpha_{1}, j}^{\alpha_{0}}$, which follows from (6.14), we obtain

$$
R_{\alpha_{0}}^{i}\left(Z_{\alpha_{1}, j}^{\alpha_{0}} R_{\beta_{0}}^{j}+F_{\beta_{0} \gamma_{0}}^{\alpha_{0}} Z_{\alpha_{1}}^{\gamma_{0}}\right)=0
$$

Again, this may be solved by introducing additional structure tensors $G_{\beta_{0} \alpha_{1}}^{\gamma_{1}}(A)$

$$
Z_{\alpha_{1}, j}^{\alpha_{0}} R_{\beta_{0}}^{j}+F_{\beta_{0} \gamma_{0}}^{\alpha_{0}} Z_{\alpha_{1}}^{\gamma_{0}}=-Z_{\gamma_{1}}^{\alpha_{0}} G_{\beta_{0} \alpha_{1}}^{\gamma_{1}},
$$

thus defining a new structure equation for first-stage reducible theories. Multiplying this equation by $Z_{\beta_{1}}^{\beta_{0}}$ and taking into account (6.14),

$$
F_{\beta_{0} \gamma_{0}}^{\alpha_{0}} Z_{\alpha_{1}}^{\gamma_{0}} Z_{\beta_{1}}^{\beta_{0}}=-Z_{\gamma_{1}}^{\alpha_{0}} Z_{\beta_{1}}^{\beta_{0}} G_{\beta_{0} \alpha_{1}}^{\gamma_{1}}
$$

we obtain the useful equality

$$
Z_{\beta_{1}}^{\alpha_{0}} G_{\alpha_{0} \alpha_{1}}^{\gamma_{1}}=-Z_{\alpha_{1}}^{\alpha_{0}} G_{\alpha_{0} \beta_{1}}^{\gamma_{1}}
$$

Moreover, we are able to establish two further gauge structure relations for the firststage reducible case showing that $H_{\alpha_{0} \beta_{0} \gamma_{0}}^{\alpha_{1}}$ and $G_{\alpha_{0} \beta_{1}}^{\alpha_{1}}$ are not independent of each other. The first one reads

$$
\left(G_{\beta_{0} \gamma_{1}}^{\alpha_{1}} G_{\gamma_{0} \beta_{1}}^{\gamma_{1}}+R_{\beta_{0}}^{i} G_{\gamma_{0} \beta_{1}, i}^{\alpha_{1}}+\operatorname{antisym}\left(\beta_{0} \leftrightarrow \gamma_{0}\right)\right)+G_{\alpha_{0} \beta_{1}}^{\alpha_{1}} F_{\beta_{0} \gamma_{0}}^{\alpha_{0}}+3 Z_{\beta_{1}}^{\alpha_{0}} H_{\alpha_{0} \beta_{0} \gamma_{0}}^{\alpha_{1}}=0 .
$$

In order to verify this relation we multiply the Jacobi identity (6.17) with $Z_{\beta_{1}}^{\alpha_{0}}$. By virtue of $R_{\alpha_{0}}^{i} Z_{\beta_{1}}^{\alpha_{0}}=0$ this yields

$$
\begin{aligned}
& \left(F_{\eta_{0} \alpha_{0}}^{\delta_{0}} Z_{\beta_{1}}^{\alpha_{0}}\right) F_{\beta_{0} \gamma_{0}}^{\eta_{0}}+F_{\eta_{0} \beta_{0}}^{\delta_{0}}\left(F_{\gamma_{0} \alpha_{0}}^{\eta_{0}} Z_{\beta_{1}}^{\alpha_{0}}\right)-F_{\eta_{0} \gamma_{0}}^{\delta_{0}}\left(F_{\beta_{0} \alpha_{0}}^{\eta_{0}} Z_{\beta_{1}}^{\alpha_{0}}\right) \\
& \quad-R_{\beta_{0}}^{i}\left(F_{\gamma_{0} \alpha_{0}, i}^{\delta_{0}} Z_{\beta_{1}}^{\alpha_{0}}\right)+R_{\gamma_{0}}^{i}\left(F_{\beta_{0} \alpha_{0}, i}^{\delta_{0}} Z_{\beta_{1}}^{\alpha_{0}}\right)-Z_{\alpha_{1}}^{\delta_{0}}\left(3 Z_{\beta_{1}}^{\alpha_{0}} H_{\alpha_{0} \beta_{0} \gamma_{0}}^{\alpha_{1}}\right)=0 .
\end{aligned}
$$


After replacing all terms of the form $F_{\eta_{0} \alpha_{0}}^{\delta_{0}} Z_{\beta_{1}}^{\alpha_{0}}$ according to (6.18) this gives

$$
\begin{aligned}
& Z_{\beta_{1}, i}^{\delta_{0}}\left(R_{\alpha_{0}}^{i} F_{\beta_{0} \gamma_{0}}^{\alpha_{0}}\right)+Z_{\alpha_{1}}^{\delta_{0}}\left(G_{\alpha_{0} \beta_{1}}^{\alpha_{1}} F_{\beta_{0} \gamma_{0}}^{\alpha_{0}}+3 Z_{\beta_{1}}^{\alpha_{0}} H_{\alpha_{0} \beta_{0} \gamma_{0}}^{\alpha_{1}}\right) \\
& +\left\{R_{\beta_{0}}^{i}\left(F_{\gamma_{0} \alpha_{0}, i}^{\delta_{0}} Z_{\beta_{1}}^{\alpha_{0}}-F_{\alpha_{0} \gamma_{0}}^{\delta_{0}} Z_{\beta_{1}, i}^{\alpha_{0}}\right)-\left(F_{\alpha_{0} \gamma_{0}}^{\delta_{0}} Z_{\alpha_{1}}^{\alpha_{0}}\right) G_{\beta_{0} \beta_{1}}^{\alpha_{1}}+\operatorname{antisym}\left(\beta_{0} \leftrightarrow \gamma_{0}\right)\right\}=0,
\end{aligned}
$$

and, using the same relation once more,

$$
\begin{aligned}
& Z_{\beta_{1}, i}^{\delta_{0}}\left(R_{\alpha_{0}}^{i} F_{\beta_{0} \gamma_{0}}^{\alpha_{0}}\right)+Z_{\alpha_{1}}^{\delta_{0}}\left(G_{\alpha_{0} \beta_{1}}^{\alpha_{1}} F_{\beta_{0} \gamma_{0}}^{\alpha_{0}}+3 Z_{\beta_{1}}^{\alpha_{0}} H_{\alpha_{0} \beta_{0} \gamma_{0}}^{\alpha_{1}}\right) \\
& +\left\{R_{\beta_{0}}^{i}\left(\left(F_{\gamma_{0} \alpha_{0}}^{\delta_{0}} Z_{\beta_{1}}^{\alpha_{0}}\right)_{, i}+Z_{\alpha_{1}, i}^{\delta_{0}} G_{\gamma_{0} \beta_{1}}^{\alpha_{1}}\right)+Z_{\alpha_{1}}^{\delta_{0}} G_{\beta_{0} \gamma_{1}}^{\alpha_{1}} G_{\gamma_{0} \beta_{1}}^{\gamma_{1}}+\operatorname{antisym}\left(\beta_{0} \leftrightarrow \gamma_{0}\right)\right\}=0 .
\end{aligned}
$$

Here, the expression in the curly bracket can be rewritten as

$$
\begin{aligned}
& Z_{\beta_{1}, i}^{\delta_{0}}\left(R_{\alpha_{0}}^{i} F_{\beta_{0} \gamma_{0}}^{\alpha_{0}}\right)+Z_{\alpha_{1}}^{\delta_{0}}\left(G_{\alpha_{0} \beta_{1}}^{\alpha_{1}} F_{\beta_{0} \gamma_{0}}^{\alpha_{0}}+3 Z_{\beta_{1}}^{\alpha_{0}} H_{\alpha_{0} \beta_{0} \gamma_{0}}^{\alpha_{1}}\right) \\
& +\left\{R_{\beta_{0}}^{i}\left(F_{\gamma_{0} \alpha_{0}}^{\delta_{0}} Z_{\beta_{1}}^{\alpha_{0}}+Z_{\alpha_{1}}^{\delta_{0}} G_{\gamma_{0} \beta_{1}}^{\alpha_{1}}\right)_{, i}+Z_{\alpha_{1}}^{\delta_{0}}\left(G_{\gamma_{1} \beta_{0}}^{\alpha_{1}} G_{\gamma_{0} \beta_{1}}^{\gamma_{1}}+R_{\gamma_{0}}^{i} G_{\beta_{0} \beta_{1}, i}^{\alpha_{1}}\right)+\operatorname{antisym}\left(\beta_{0} \leftrightarrow \gamma_{0}\right)\right\}=0
\end{aligned}
$$

and furthermore, once again using relation (6.18),

$$
\begin{aligned}
& Z_{\beta_{1}, i}^{\delta_{0}}\left(R_{\alpha_{0}}^{i} F_{\beta_{0} \gamma_{0}}^{\alpha_{0}}\right)+Z_{\alpha_{1}}^{\delta_{0}}\left(G_{\alpha_{0} \beta_{1}}^{\alpha_{1}} F_{\beta_{0} \gamma_{0}}^{\alpha_{0}}+3 Z_{\beta_{1}}^{\alpha_{0}} H_{\alpha_{0} \beta_{0} \gamma_{0}}^{\alpha_{1}}\right) \\
& -\left\{R_{\beta_{0}}^{i}\left(Z_{\beta_{1}, j}^{\delta_{0}} R_{\gamma_{0}, i}^{j}\right)_{, i}-Z_{\alpha_{1}}^{\delta_{0}}\left(G_{\beta_{0} \gamma_{1}}^{\alpha_{1}} G_{\gamma_{0} \beta_{1}}^{\gamma_{1}}+R_{\gamma_{0}}^{i} G_{\beta_{0} \beta_{1}, i}^{\alpha_{1}}\right)+\operatorname{antisym}\left(\beta_{0} \leftrightarrow \gamma_{0}\right)\right\}=0 .
\end{aligned}
$$

This equation, since the algebra (6.15) is closed,

$$
Z_{\beta_{1}, i}^{\delta_{0}}\left(R_{\alpha_{0}}^{i} F_{\beta_{0} \gamma_{0}}^{\alpha_{0}}\right)=Z_{\beta_{1}, i}^{\delta_{0}}\left(R_{\beta_{0}}^{j} R_{\gamma_{0}, j}^{i}-R_{\gamma_{0}}^{j} R_{\beta_{0}, j}^{i}\right)=R_{\beta_{0}}^{i}\left(Z_{\beta_{1}, j}^{\delta_{0}} R_{\gamma_{0}}^{j}\right)_{, i}-R_{\gamma_{0}}^{i}\left(Z_{\beta_{1}, j}^{\delta_{0}} R_{\beta_{0}}^{j}\right)_{, i},
$$

leads immediately to the gauge structure relation (6.20).

The second gauge structure relation, which can also be derived by means of the Jacobi identity, is given by

$$
\begin{aligned}
& \left(H_{\eta_{0} \alpha_{0} \beta_{0}}^{\alpha_{1}} F_{\gamma_{0} \delta_{0}}^{\eta_{0}}-H_{\eta_{0} \delta_{0} \alpha_{0}}^{\alpha_{1}} F_{\beta_{0} \gamma_{0}}^{\eta_{0}}+\operatorname{cyclic} \operatorname{perm}\left(\alpha_{0}, \beta_{0}, \gamma_{0}\right)\right) \\
& +\left\{R_{\delta_{0}}^{i} H_{\alpha_{0} \beta_{0} \gamma_{0}, i}^{\alpha_{1}}-G_{\delta_{0} \beta_{1}}^{\alpha_{1}} H_{\alpha_{0} \beta_{0} \gamma_{0}}^{\beta_{1}}+\operatorname{antisym}\left(\delta_{0} \leftrightarrow\left(\alpha_{0}, \beta_{0}, \gamma_{0}\right)\right)\right\}=0,
\end{aligned}
$$

where the left-hand side is a totally antisymmetric expression with respect to $\left(\alpha_{0}, \beta_{0}, \gamma_{0}, \delta_{0}\right)$.

In order to prove that this relation is satisfied we consider the following identity:

$$
\begin{aligned}
& \left\{\left(\left(Z_{\alpha_{1}}^{\lambda_{0}} H_{\eta_{0} \alpha_{0} \beta_{0}}^{\alpha_{1}}\right) F_{\gamma_{0} \delta_{0}}^{\eta_{0}}+\text { cyclic perm }\left(\alpha_{0}, \beta_{0}, \gamma_{0}\right)\right)\right. \\
& \left.\quad+2 R_{\delta_{0}}^{i}\left(Z_{\alpha_{1}}^{\lambda_{0}} H_{\alpha_{0} \beta_{0} \gamma_{0}}^{\alpha_{1}}\right)_{, i}+2 F_{\delta_{0} \eta_{0}}^{\lambda_{0}}\left(Z_{\alpha_{1}}^{\eta_{0}} H_{\alpha_{0} \beta_{0} \gamma_{0}}^{\alpha_{1}}\right)\right\}+\operatorname{antisym}\left(\delta_{0} \leftrightarrow\left(\alpha_{0}, \beta_{0}, \gamma_{0}\right)\right) \equiv 0
\end{aligned}
$$

which can be verified by a direct calculation replacing the terms $Z_{\alpha_{1}}^{\lambda_{0}} H_{\alpha_{0} \beta_{0} \gamma_{0}}^{\alpha_{1}}$ by the help of the Jacobi identity (6.17). Taking into account (6.18) one obtains the equation

$$
\begin{aligned}
Z_{\alpha_{1}}^{\lambda_{0}}\{ & \left(H_{\eta_{0} \alpha_{0} \beta_{0}}^{\alpha_{1}} F_{\gamma_{0} \delta_{0}}^{\eta_{0}}+\text { cyclic perm }\left(\alpha_{0}, \beta_{0}, \gamma_{0}\right)\right) \\
& \left.+2 R_{\delta_{0}}^{i} H_{\alpha_{0} \beta_{0} \gamma_{0}, i}^{\alpha_{1}}-2 G_{\beta_{1} \delta_{0}}^{\alpha_{1}} H_{\alpha_{0} \beta_{0} \gamma_{0}}^{\beta_{1}}\right\}+\operatorname{antisym}\left(\delta_{0} \leftrightarrow\left(\alpha_{0}, \beta_{0}, \gamma_{0}\right)\right)=0 .
\end{aligned}
$$


After factoring out the zero-modes $Z_{\alpha_{1}}^{\lambda_{0}}$ and using the identity

$$
\begin{aligned}
& \left(H_{\eta_{0} \alpha_{0} \beta_{0}}^{\alpha_{1}} F_{\gamma_{0} \delta_{0}}^{\eta_{0}}+\text { cyclic perm }\left(\alpha_{0}, \beta_{0}, \gamma_{0}\right)\right)+\operatorname{antisym}\left(\delta_{0} \leftrightarrow\left(\alpha_{0}, \beta_{0}, \gamma_{0}\right)\right) \\
& \equiv 2\left(H_{\eta_{0} \alpha_{0} \beta_{0}}^{\alpha_{1}} F_{\gamma_{0} \delta_{0}}^{\eta_{0}}-H_{\eta_{0} \delta_{0} \alpha_{0}}^{\alpha_{1}} F_{\beta_{0} \gamma_{0}}^{\eta_{0}}+\operatorname{cyclic} \operatorname{perm}\left(\alpha_{0}, \beta_{0}, \gamma_{0}\right)\right)
\end{aligned}
$$

this equation acquires the form (6.22). The relations $(6.13)-(6.22)$ are the key equations in order to derive the $\operatorname{sl}(1,2)$-transformations of the fields for the first-stage reducible case.

In order to construct the proper solution $S_{m}=S_{\mathrm{cl}}+\left(\frac{1}{2} \epsilon_{a b} \mathbf{s}_{m}^{b} \mathbf{s}_{m}^{a}+m^{2}\right) X$ in that case one has to choose $X=\bar{A}_{i} A^{i}+\bar{B}_{\alpha_{0}} B^{\alpha_{0}}+\bar{B}_{\alpha_{1} a} B^{\alpha_{1} a}+\bar{C}_{\alpha_{0} a} C^{\alpha_{0} a}+\bar{C}_{\alpha_{1} a b} C^{\alpha_{1} a b}$. A realization of the $s l(1,2)$-transformations of the antifields $\bar{A}_{i}, \bar{B}_{\alpha_{0}}, \bar{B}_{\alpha_{1} a}, \bar{C}_{\alpha_{0} a}$ and $\bar{C}_{\alpha_{1} a b}$ already has been given (see Appendix A). The corresponding nonlinear realization of the $\operatorname{sl}(1,2)$ in terms of the fields $A^{i}, B^{\alpha_{0}}, B^{\alpha_{1} a}, C^{\alpha_{0} a}$ and $C^{\alpha_{1} a b}$ are the following

(1) translations:

$$
\begin{aligned}
\mathbf{s}_{+}^{a} A^{i}= & R_{\alpha_{0}}^{i} C^{\alpha_{0} a} \\
\mathbf{s}_{+}^{a} C^{\alpha_{0} b}= & Z_{\alpha_{1}}^{\alpha_{0}} C^{\alpha_{1} a b}+\epsilon^{a b} B^{\alpha_{0}}-F_{\beta_{0} \gamma_{0}}^{\alpha_{0}} C^{\beta_{0} a} C^{\gamma_{0} b}, \\
\mathbf{s}_{+}^{a} B^{\alpha_{0}}= & Z_{\alpha_{1}}^{\alpha_{0}} B^{\alpha_{1} a}+\frac{1}{2} F_{\beta_{0} \gamma_{0}}^{\alpha_{0}}\left(B^{\beta_{0}} C^{\gamma_{0} a}-\epsilon_{c d} Z_{\alpha_{1}}^{\beta_{0}} C^{\alpha_{1} a c} C^{\gamma_{0} d}\right) \\
& +\frac{1}{12} \epsilon_{c d}\left(F_{\eta_{0} \beta_{0}}^{\alpha_{0}} F_{\gamma_{0} \delta_{0}}^{\eta_{0}}+2 R_{\beta_{0}}^{i} F_{\gamma_{0} \delta_{0}, i}^{\alpha_{0}}\right) C^{\gamma_{0} a} C^{\delta_{0} c} C^{\beta_{0} d}, \\
\mathbf{s}_{+}^{a} C^{\alpha_{1} b c}= & -\epsilon^{a c} B^{\alpha_{1} b}-\epsilon^{a b} B^{\alpha_{1} c}+G_{\alpha_{0} \beta_{1}}^{\alpha_{1}} C^{\alpha_{0} a} C^{\beta_{1} b c}-\frac{1}{2} H_{\alpha_{0} \beta_{0} \gamma_{0}}^{\alpha_{1}} C^{\alpha_{0} a} C^{\beta_{0} b} C^{\gamma_{0} c}, \\
\mathbf{s}_{+}^{a} B^{\alpha_{1} b}= & G_{\alpha_{0} \beta_{1}}^{\alpha_{1}}\left(C^{\alpha_{0} a} B^{\beta_{1} b}-\frac{1}{2} \epsilon_{c d} Z_{\gamma_{1}}^{\alpha_{0}} C^{\gamma_{1} a c} C^{\beta_{1} b d}\right)-\frac{1}{2} H_{\alpha_{0} \beta_{0} \gamma_{0}}^{\alpha_{1}} B^{\alpha_{0}} C^{\beta_{0} a} C^{\gamma_{0} c} \\
& +\frac{1}{4} \epsilon_{c d} H_{\alpha_{0} \beta_{0} \gamma_{0}}^{\alpha_{1}} Z_{\beta_{1}}^{\alpha_{0}}\left(3 C^{\beta_{0} a} C^{\beta_{1} b c} C^{\gamma_{0} d}+C^{\beta_{0} b} C^{\beta_{1} a c} C^{\gamma_{0} d}\right) \\
& +\frac{1}{8} \epsilon_{c d}\left(G_{\delta_{0} \beta_{1}}^{\alpha_{1}} H_{\alpha_{0} \beta_{0} \gamma_{0}}^{\beta_{1}}-R_{\delta_{0}}^{i} H_{\alpha_{0} \beta_{0} \gamma_{0}, i}^{\alpha_{1}}\right) C^{\gamma_{0} a} C^{\beta_{0} b} C^{\alpha_{0} c} C^{\delta_{0} d} \\
& -\frac{1}{16} \epsilon_{c d} H_{\eta_{0} \alpha_{0} \beta_{0}}^{\alpha_{1}} F_{\gamma_{0} \delta_{0}}^{\eta_{0}}\left(C^{\gamma_{0} a} C^{\beta_{0} b}+C^{\gamma_{0} b} C^{\beta_{0} a}\right) C^{\alpha_{0} c} C^{\delta_{0} d},
\end{aligned}
$$

(2) special conformal transformations:

$$
\begin{aligned}
\mathbf{s}_{-}^{a} A^{i} & =0, \\
\mathbf{s}_{-}^{a} C^{\alpha_{0} b} & =0, \\
\mathbf{s}_{-}^{a} B^{\alpha_{0}} & =-2 C^{\alpha_{0} a}, \\
\mathbf{s}_{-}^{a} C^{\alpha_{1} b c} & =0, \\
\mathbf{s}_{-}^{a} B^{\alpha_{1} b} & =2 C^{\alpha_{1} a b},
\end{aligned}
$$


(3) symplectic rotations:

$$
\begin{aligned}
\mathbf{d}_{\alpha} A^{i} & =0 \\
\mathbf{d}_{\alpha} C^{\alpha_{0} b} & =C^{\alpha_{0} a}\left(\sigma_{\alpha}\right)_{a}{ }^{b}, \\
\mathbf{d}_{\alpha} B^{\alpha_{0}} & =0 \\
\mathbf{d}_{\alpha} C^{\alpha_{1} b c} & =C^{\alpha_{1} a c}\left(\sigma_{\alpha}\right)_{a}^{b}+C^{\alpha_{1} b a}\left(\sigma_{\alpha}\right)_{a}^{c}, \\
\mathbf{d}_{\alpha} B^{\alpha_{1} b} & =B^{\alpha_{1} a}\left(\sigma_{\alpha}\right)_{a}{ }^{b},
\end{aligned}
$$

and (4) dilatations:

$$
\begin{aligned}
\mathbf{d} A^{i} & =0 \\
\mathbf{d} C^{\alpha_{0} b} & =C^{\alpha_{0} b}, \\
\mathbf{d} B^{\alpha_{0}} & =2 B^{\alpha_{0}}, \\
\mathbf{d} C^{\alpha_{1} b c} & =2 C^{\alpha_{1} b c}, \\
\mathbf{d} B^{\alpha_{1} b} & =3 B^{\alpha_{1} b} .
\end{aligned}
$$

By making use of Eqs. (6.13)-(6.22) after somewhat involved and tedious algebraic manipulations it can be proven that the transformations (6.23)-(6.26) really obey the $\operatorname{sl}(1,2)-$ superalgebra (6.4). For some details of this work we refer to Ref. [8] where similar calculations were performed for the $\operatorname{osp}(1,2)$-superalgebra.

Continuing in the same way, analogous considerations can be made for higher stage reducible theories. But then, more and more new gauge structure tensors with increasing numbers of indices and additional gauge structure relations appear which makes a study of these theories quite complicated.

\section{Concluding remarks}

In this paper we have revealed the geometrical content of the $\operatorname{osp}(1,2)$-covariant Lagrangian quantization of general massive gauge theories. A natural geometric formulation of that quantization procedure is obtained by considering $\operatorname{osp}(1,2)$ as subsuperalgebra of $s l(1,2)$, which is considered as the algebra of generators of conformal transformations in two anticommuting dimensions. It is shown that proper solutions of the classical master equations can be constructed being invariant under $\operatorname{osp}(1,2) \oplus u(1)$. The $m$-dependent extended BRST symmetry is realized in superspace as translations combined with $m^{-}$ dependent special conformal transformations. The $s l(2) \oplus u(1)$ symmetry is realized in 
superspace as symplectic rotations and dilatations, respectively. By the choice of a gauge the $\operatorname{sl}(2) \oplus u(1)$ symmetry is broken down to $\operatorname{sl}(2) \sim \operatorname{sp}(2)$. In principle, by formal manipulations it is also possible to construct proper solutions of the corresponding quantum master equations. However, in doing so a serious problem is to provide a sensible definition of the various $\Delta$-operators of the quantum master equations, which do not make sense when applied to local functionals. In this paper we have not adressed such problems and related questions as the use of explicit regularizations and renormalizations schemes and the discussion of the role of anomalies.

\section{Acknowledgement}

The authors would like to thank P.M. Lavrov for valuable discussions concerning various aspects of the superfield quantization of general gauge theories.

\section{A Componentwise notation of the $\operatorname{sl}(1,2)$ transforma- tions of the antifields}

In componentwise notation the linear transformations (2.7) generated by $V_{+}^{a}$ and $V_{-}^{a}$ read as follows:

$$
\begin{aligned}
V_{+}^{a} \bar{A}_{i} & =\epsilon^{a b} A_{i b}^{*}, \\
V_{+}^{a} A_{i b}^{*} & =-\delta_{b}^{a} D_{i}, \\
V_{+}^{a} D_{i} & =0 \\
V_{+}^{a} \bar{B}_{\alpha_{s} \mid a_{1} \cdots a_{s}} & =\epsilon^{a b} B_{\alpha_{s} b \mid a_{1} \cdots a_{s}}^{*}, \\
V_{+}^{a} B_{\alpha_{s} b \mid a_{1} \cdots a_{s}}^{*} & =-\delta_{b}^{a} E_{\alpha_{s} \mid a_{1} \cdots a_{s}}, \\
V_{+}^{a} E_{\alpha_{s} \mid a_{1} \cdots a_{s}} & =0, \\
V_{+}^{a} \bar{C}_{\alpha_{s} \mid a_{0} \cdots a_{s}} & =\epsilon^{a b} C_{\alpha_{s} b \mid a_{0} \cdots a_{s}}^{*}, \\
V_{+}^{a} C_{\alpha_{s} b \mid a_{0} \cdots a_{s}}^{*} & =-\delta_{b}^{a} F_{\alpha_{s} \mid a_{0} \cdots a_{s}}, \\
V_{+}^{a} F_{\alpha_{s} \mid a_{0} \cdots a_{s}} & =0
\end{aligned}
$$


and

$$
\begin{aligned}
V_{-}^{a} \bar{A}_{i} & =0 \\
V_{-}^{a} A_{i b}^{*} & =2 \delta_{b}^{a} \bar{A}_{i}, \\
V_{-}^{a} D_{i} & =0 \\
V_{-}^{a} \bar{B}_{\alpha_{s} \mid a_{1} \cdots a_{s}} & =0 \\
V_{-}^{a} B_{\alpha_{s} b \mid a_{1} \cdots a_{s}}^{*} & =2 \delta_{b}^{a}\left(\bar{B}_{\alpha_{s} \mid a_{1} \cdots a_{s}}+\sum_{r=1}^{s} \delta_{a_{r}}^{a} \bar{B}_{\alpha_{s} \mid a_{1} \cdots a_{r-1} b a_{r+1} \cdots a_{s}}\right), \\
V_{-}^{a} E_{\alpha_{s} \mid a_{1} \cdots a_{s}} & =2 \epsilon^{a b}\left((s+2) B_{\alpha_{s} b \mid a_{1} \cdots a_{s}}-\sum_{r=1}^{s} B_{\alpha_{s} a_{r} \mid a_{1} \cdots a_{r-1} b a_{r+1} \cdots a_{s}}^{*}\right), \\
V_{-}^{a} \bar{C}_{\alpha_{s} \mid a_{0} \cdots a_{s}} & =0 \\
V_{-}^{a} C_{\alpha_{s} b \mid a_{0} \cdots a_{s}}^{*} & =2 \delta_{b}^{a}\left(\bar{C}_{\alpha_{s} \mid a_{0} \cdots a_{s}}+\sum_{r=0}^{s} \delta_{a_{r}}^{a} \bar{C}_{\alpha_{s} \mid a_{0} \cdots a_{r-1} b a_{r+1} \cdots a_{s}}\right), \\
V_{-}^{a} F_{\alpha_{s} \mid a_{0} \cdots a_{s}} & =2 \epsilon^{a b}\left((s+1) C_{\alpha_{s} b \mid a_{0} \cdots a_{s}}-\sum_{r=0}^{s} C_{\alpha_{s} a_{r} \mid a_{0} \cdots a_{r-1} b a_{r+1} \cdots a_{s}}^{*}\right),
\end{aligned}
$$

where the definitions (2.9) and (2.11) have to be taken into account. For the transformations (2.8) generated by $V_{\alpha}$ and $V$ one gets:

$$
\begin{aligned}
V_{\alpha} \bar{A}_{i} & =0 \\
V_{\alpha} A_{i b}^{*} & =A_{i a}^{*}\left(\sigma_{\alpha}\right)_{b}^{a}, \\
V_{\alpha} D_{i} & =0 \\
V_{\alpha} \bar{B}_{\alpha_{s} \mid a_{1} \cdots a_{s}} & =\sum_{r=1}^{s} \bar{B}_{\alpha_{s} \mid a_{1} \cdots a_{r-1} a a_{r+1} \cdots a_{s}}\left(\sigma_{\alpha}\right)^{a}{ }_{a_{r}}, \\
V_{\alpha} B_{\alpha_{s} b \mid a_{1} \cdots a_{s}}^{*} & =B_{\alpha_{s} a \mid a_{1} \cdots a_{s}}^{*}\left(\sigma_{\alpha}\right)^{a}{ }_{b}+\sum_{r=1}^{s} B_{\alpha_{s} b \mid a_{1} \cdots a_{r-1} a a_{r+1} \cdots a_{s}}^{*}\left(\sigma_{\alpha}\right)^{a} a_{r}, \\
V_{\alpha} E_{\alpha_{s} \mid a_{1} \cdots a_{s}} & =\sum_{r=1}^{s} E_{\alpha_{s} \mid a_{1} \cdots a_{r-1} a a_{r+1} \cdots a_{s}}\left(\sigma_{\alpha}\right)^{a}{ }_{a_{r}}, \\
V_{\alpha} \bar{C}_{\alpha_{s} \mid a_{0} \cdots a_{s}} & =\sum_{r=0}^{s} \bar{C}_{\alpha_{s} \mid a_{0} \cdots a_{r-1} a a_{r+1} \cdots a_{s}}\left(\sigma_{\alpha}\right)^{a}{ }_{a_{r}}, \\
V_{\alpha} C_{\alpha_{s} b \mid a_{0} \cdots a_{s}}^{*} & =C_{\alpha_{s} a \mid a_{0} \cdots a_{s}}^{*}\left(\sigma_{\alpha}\right)_{b}^{a}+\sum_{r=0}^{s} C_{\alpha_{s} b \mid a_{0} \cdots a_{r-1} a a_{r+1} \cdots a_{s}}\left(\sigma_{\alpha}\right)^{a}{ }_{a_{r}}, \\
V_{\alpha} F_{\alpha_{s} \mid a_{0} \cdots a_{s}} & =\sum_{r=0}^{s} F_{\alpha_{s} \mid a_{0} \cdots a_{r-1} a a_{r+1} \cdots a_{s}}\left(\sigma_{\alpha}\right)^{a}{ }_{a_{r}}
\end{aligned}
$$


and

$$
\begin{aligned}
V \bar{A}_{i} & =-2 \bar{A}_{i}, \\
V A_{i b}^{*} & =-3 A_{i b}^{*}, \\
V D_{i} & =-4 D_{i}, \\
V \bar{B}_{\alpha_{s} \mid a_{1} \cdots a_{s}} & =-(s+4) \bar{B}_{\alpha_{s} \mid a_{1} \cdots a_{s}}, \\
V B_{\alpha_{s} \mid a_{1} \cdots a_{s}}^{*} & =-(s+5) B_{\alpha_{s} \mid a_{1} \cdots a_{s}}^{*}, \\
V E_{\alpha_{s} \mid a_{0} \cdots a_{s}} & =-(s+6) E_{\alpha_{s} \mid a_{0} \cdots a_{s}}, \\
V \bar{C}_{\alpha_{s} \mid a_{0} \cdots a_{s}} & =-(s+3) \bar{C}_{\alpha_{s} \mid a_{1} \cdots a_{s}}, \\
V C_{\alpha_{s} \mid a_{0} \cdots a_{s}}^{*} & =-(s+4) C_{\alpha_{s} \mid a_{0} \cdots a_{s}}^{*}, \\
V F_{\alpha_{s} \mid a_{0} \cdots a_{s}} & =-(s+5) F_{\alpha_{s} \mid a_{0} \cdots a_{s}} .
\end{aligned}
$$

By an explicit calculation it can be verified that the generators $V_{ \pm}^{a}, V_{\alpha}$ and $V$ obey the $\operatorname{sl}(1,2)$-superalgebra (2.3).

\section{References}

[1] C. Becchi, A. Rouet and R. Stora, Phys. Lett. B 52 (1974) 344; I.V. Tyutin, Gauge invariance in field theory and statistical physics in operator formalism, Lebedev preprint FIAN 39 (1979), unpublished;

[2] G. Curci and R. Ferrari, Phys. Lett. B 63 (1976) 91; I. Ojima, Prog. Theor. Phys. $64(1980) 625$

[3] S. Weinberg, The Quantum Theory of Fields, Vol.II, Ch. 15, Cambridge University Press, 1996

[4] L. Bonora and M. Tonin, Phys. Lett. B 98 (1981) 48

[5] C.M. Hull, B. Spence and J.L. Vazquez-Bello, Nucl. Phys. B 348 (1991) 108

[6] P.M. Lavrov, Phys. Lett. B 366 (1996) 160

[7] I.A. Batalin, K. Bering and P.H. Damgaard, Nucl. Phys. B 515 (1998) 455

[8] B. Geyer, P.M. Lavrov and D. Mülsch, J. Math. Phys. 40 (1999) 674;

B. Geyer, P.M. Lavrov and D. Mülsch, J. Math. Phys. 40 (1999) 6189 
[9] for a review see: O. Piguet and A. Rouet Phys. Repts. 76 (1981) 1; O. Piguet and S.P. Sorella , Algebraic renormalization, Springer, Berlin 1995

[10] F. De Jonghe, J. Paris and W. Troost, Nucl. Phys. B 476 (1996) 559

[11] W. Troost, P. van Nieuwenhuizen and A. Van Proeyen, Nucl. Phys. B 333 (1990) 727

[12] J. Paris, Nucl. Phys. B 450 (1995) 357; J. Paris and W. Troost, Nucl. Phys. B 482 (1996) 373

[13] I.A. Batalin, P.M. Lavrov and I.V. Tyutin, J. Math. Phys. 31 (1990) 1487; I.A. Batalin, P.M. Lavrov and I.V. Tyutin, J. Math. Phys. 32 (1991) 532

[14] L. Baulieu, W. Siegel and B. Zwiebach, Nucl. Phys. B 287 (1987) 93

[15] B.S. De Witt, Dynamical Theory of Groups and Fields (Gordan and Breach, New York, 1965)

[16] I.A. Batalin and G.A. Vilkovisky, Phys. Lett. B 102 (1981) 27

[17] I.A. Batalin and G.A. Vilkovisky, Phys. Rev. D 28 (1983) 2567

[18] M. Scheunert, W. Nahm and V. Rittenberg, J. Math. Phys. 18 (1976) 146, 155; see also: L. Frappat, P. Sorba and S. Sciarrino, Dictionary on Lie Superalgebras, ENSLAPP-AL-600/96, hep-th/9607161

[19] I.A. Batalin, R. Marnelius and A.M. Semikhatov, Nucl. Phys. B 446 (1995) 249; I.A. Batalin and R. Marnelius, Phys. Lett. B 98 (1995) 44 